\title{
Global Dynamics of a Multi-group SEIR Epidemic Model with Infection Age*
}

\author{
Vijay Pal BAJIYA ${ }^{1}$ Jai Prakash TRIPATHI ${ }^{1}$ Vipul KAKKAR ${ }^{1}$ \\ Jinshan WANG ${ }^{2}$ Guiquan $\mathrm{SUN}^{3}$
}

\begin{abstract}
Consider the heterogeneity (e.g., heterogeneous social behaviour, heterogeneity due to different geography, contrasting contact patterns and different numbers of sexual partners etc.) of host population, in this paper, the authors propose an infection age multigroup SEIR epidemic model. The model system also incorporates the feedback variables, where the infectivity of infected individuals may depend on the infection age. In the direction of mathematical analysis of model, the basic reproduction number $R_{0}$ has been computed. The global stability of disease-free equilibrium and endemic equilibrium have been established in the term of $R_{0}$. More precisely, for $R_{0} \leq 1$, the disease-free equilibrium is globally asymptotically stable and for $R_{0}>1$, they establish global stability of endemic equilibrium using some graph theoretic techniques to Lyapunov function method. By considering a numerical example, they investigate the effects of infection age and feedback on the prevalence of the disease. Their result shows that feedback parameters have different and even opposite effects on different groups. However, by choosing an appropriate value of feedback parameters, the disease could be eradicated or maintained at endemic level. Besides, the infection age of infected individuals may also change the behaviour of the disease, global stable to damped oscillations or damped oscillations to global stable.
\end{abstract}

Keywords Multi-group model, Infection age, Feedback, Graph-theoretic approach, Lyapunov function

2000 MR Subject Classification 94C15, 49K15, 49K20, 92D25, 34Dxx, 35Pxx, 920B5

\section{Introduction}

Mathematical Modelling in Epidemiology: Over the past several years, human health have continuously been threatened and thousands of people died of various infectious diseases every year (see [1-3]). By the World Health Report 1996 (WHO for short) (see [4]): "Nearly

Manuscript received May 16, 2020. Revised March 11, 2021.

${ }^{1}$ Department of Mathematics, Central University of Rajasthan, Bandar Sindri-305817, Kishangarh,

Ajmer, Rajasthan, India.

E-mail: 2017phdmt05@curaj.ac.in; jtripathi85@gmail.com; vipulk@curaj.ac.in

${ }^{2}$ Complex Systems Research Center, Shanxi University, Taiyuan 030006, China.

E-mail: wangjinshanwe@163.com

${ }^{3}$ Corresponding author. Department of Mathematics, North University of China, Taiyuan 030051, China;

Complex Systems Research Center, Shanxi University, Taiyuan 030006, China.

E-mail: gquansun@126.com

* This work was supported by the National Natural Science Foundation of China (No. 12022113), Henry Fok Foundation for Young Teachers, China (No.171002), Outstanding Young Talents Support Plan of Shanxi Province, Science and Engineering Research Board (SERB for short), India (No. ECR/2017/002786), UGC-BSR Research Start-Up-Grant, India (No. F.30-356/2017(BSR)), and Senior Research Fellowship from the Council of Scientific and Industrial Research (CSIR for short), India (No. 09/1131(0006)/2017-EMR-I). 
50,000 men, women and children are dying every day from infectious diseases". The report warns that on one side, several highly infectious diseases (e.g., incurable diseases, like, Ebola haemorrhagic fever, HIV/AIDS) are emerging to pose new threats; on the other hand, some of major diseases, for example, treatable and preventable diseases: Tuberculosis, Malaria and Cholera are making a deadly comeback in many parts of the world. Therefore, the study how a particular infectious disease progresses to ensure the likely outcome of an epidemic and information related to public health interventions become major concerns of public health. From last many decades, mathematical models are being used to understand the transmission dynamics, to predict the spreading patterns and future course of an outbreak and to evaluate suitable feedback strategies for various infectious diseases via relating the important factors of diseases to basic parameters of related models (see [5-7]). The Susceptible-Infected-Recovered (SIR for short) epidemic model proposed by Kermack and McKendrick [8] in the year 1927, is one of the very first compartmental models of infectious diseases. In which, they consider three compartments of homogeneous population (susceptible, infected and recovered compartments). The SIR epidemic model was very successful to capture too many observations of recorded epidemic data and also in predicting the behaviour of an outbreak. Further introducing one more compartment (exposed compartment) in SIR epidemic model, it was extend to Susceptible-Exposed-Infected-Recovered (SEIR for short) epidemic model (see [9]).

Multi-group Modelling: Heterogeneous Population: Although, while modelling simple SIRtype models, we assume the homogeneity (each individual is supposed to be similarly having random contacts) of population (see [10-13]). However, in general, during the modelling of an epidemic system, one may easily encounter an important theme i.e., population heterogeneity (in some sense, the individuals in the respective population are not similar to one another). In various aspects of disease transmission processes, one may realize heterogeneity, for instance, in case of sexually transmitted diseases, heterogeneous social behaviour, heterogeneity due to different geography, contrasting contact patterns and different number of sexual partners, different age groups with non-homogeneous susceptibility, heterogeneity in spatial distribution, heterogeneity due to multi-hosts pathogens in many diseases like West Nile Virus, subtypes of Influenza A, Plague (see [14-16]). Thus heterogeneity in host can come due to many population factors and its impact on respective epidemic dynamics has been studied in references [1718]. To understand the transmission dynamics of infectious diseases (e.g., Mumps, HIV/AIDS, Measles, Gonorrhea etc.) in an heterogeneous host population, the population may be divided into various homogeneous groups depending on different types of heterogeneity, e.g., contact patterns of individuals such as those among children and adults for Measles, or having distinct number of sexual partners for HIV/ADIS and other sexually transmitted diseases (STDs for short), age of host individuals, professions of hosts, modes of disease transmission or geographic distribution of host population such as communities, cities and countries (as in the transmission of cholera). In general, multi-group modelling in epidemiology is used to recognize the role of the heterogeneity in population. This kind of multi-group modelling also helps to model the intergroup interaction and interactions within the groups. To include irregularity of infectiousness 
of the disease agent, we divide the host population into various groups according to their epidemiological characteristics. In the existing literature of epidemiological modelling, the multi-group epidemic models have been used to investigate the transmission pattern and to describe the transmission dynamics of many infectious diseases in heterogenous population such as Cholera (see [19]), Measles, HIV/ADIS (see [20]) and Gonorrhea (see [18]). In particular, to study the impact of variations in infectiousness of HIV, Hyman et al. [20] proposed two simple models. In the first model, during the chronic phase of infection, they considered different levels of virus between individuals. The second model was based on standard hypothesis depending upon an individual current disease stage and his/her infectiousness, the infected individuals progress via a series of infection phases. The Gonorroea multi-group model is one of the earliest work in the area of multi-group epidemic modelling. This particular model was used to describe the prevalence of Gonorroea and proposed by Lajmanovich and Yorke [18]. In this particular $n$-group Susceptible-Infected-Susceptible (SIS for short) model, the authors discussed global dynamics by establishing global stability of unique endemic equilibrium, using a quadratic global Lyapunov function. Following the work of Lajmanovich and Yorke [18], Beretta and Capasso [21] studied a multi-group SIR epidemic model assuming constant population in each group. In references [18, 21], the authors discussed the global dynamics of the model by establishing sufficient conditions for global stability of endemic equilibrium. A multi-group infectious disease model with temporary immunity of recovered population was studied by Hethcote [22]. They obtained threshold criterion to determine the immunization rates relating the eradication of the disease. Afterwards, various forms of multi-group epidemic models have been discussed in [23-26]. From the above critical review of epidemic modelling of heterogeneous population, it may easily be observed that establishing the global stability of endemic equilibrium is one of the main challenges while dealing the theoretical aspect of the model.

Age of Infection in an Epidemic Model: Most of the time, we assume the homogeneity of infected individuals (i.e., all infected individuals in that class have the same epidemiological parameters) after dividing a particular host population in different classes. However, it may be a unrealistic assumption. In reality, as time progresses, the disease develops within the host individuals with the different infectivity or many times one may easily observe different infectiousness of an infected individual at different stages of infection (e.g., Cholera, Typhoid). In particular, there are diseases, whose transmissibility increases with age of infection, for example, Ebola. On the other hand, there are diseases in which the infectiousness of infected individuals increases upto a threshold level with age of infection and then starts decreasing, like Influenza (see [27]). This suggests that infectivity of host might continuously change with time and infection age may be one of the informative factor to model some of the infectious diseases. Therefore, we may consider the infectivity as the function of infection age of infected individuals. Some of the epidemic models incorporating an individual infection for some particular diseases are: Tubercolosis (see [28]), HIV/AIDS (see [29]), Chagas disease (see [30]), or pandemic Influenza (see [31]). Feng et al. [28] studied the qualitative behaviour of system of ordinary equations and system of integral-differential equations, where both models describe 
the dynamics of Tuberculosis (TB for short) disease. The authors found that the dynamical behaviour of age structured TB model (model with variable latent period) is very similar to that of TB model with ordinary differential equations. Thieme et al. [29] explored the dynamics of HIV/AIDS model incorporating long and variable periods of infectiousness, variable infectivity. They established the conditions under which the endemic equilibrium is locally stable. They found that undamped oscillations may also occur if the variable infectivity is at a higher level at certain incubation period. In particular, Rost et al. [32] studied an SEIR epidemic model with varying infectivity by considering infection age of infected individuals. Recently, McCluskey [33] proposed an SEIR epidemic model including infected individuals with infection-age structure to allow the varying infectivity. Because in the varying infectivity, the incidence term has the form $\beta S(t) \int_{0}^{\infty} k(a) i(t, a) \mathrm{d} a$, hence, Li et al. [34] extended the results of Rost et al. [32] and McCluskey [33] to a multi-group SEIR epidemic model with distributed delays. The authors discussed the global dynamics of extended model by using a graph-theoretical approach to the method of Lyapunov functionals.

Feedback in Epidemic Model: In case of many epidemics, when complete eradication of disease is not possible, then whether we may change the endemicity level of disease, becomes an important question. This means we can maintain the endemic level below a threshold value so that outbreak of disease can be avoided. In some cases, we may also want to change the endemicity of the existing equilibrium maintaining its stability. On the other hand, in real world, ecological systems are continuously disrupted by unpredictable disturbances persisting for finite period of times. The presence of such unpredictable forces may also result in alteration of various parameters like survival rate. In the language of control theory, these unpredictable forces are called the feedback variables. By introducing feedback variables, we make improvement in the associated epidemic model system which may provide us population stabilizing at lower value of equilibrium. Sometimes, the disease can also be made endemic or extinct by choosing suitable values of associated feedback variables. The understanding of the feedback technique might be implemented by means of some biological and reasonable feedback or by some harvesting mechanism (see [35]). In ecology, one important and practical issue related to feedback variables is the "ecology balance": Whether the considered ecosystem would be able to persist in the presence of such unpredictable disturbances. Indeed, in the time period of the last few decades, the dynamical behaviours of the population models with feedback have been deliberated significantly (see [36-41]). Fan et al. [42] proposed a logistic model incorporating feedback variable. They established the global stability of positive equilibrium using a new method combined with lower and upper solution technique. This method is much simple and convenient than Lyapunov function method of global stability. Yang et al. [43] studied an autonomous cooperative system with single feedback. They showed that the stable species could become die out or change its position of stable state maintaining its stability by choosing appropriate values of control parameters. An Susceptible-infected (SI for short) epidemic model with feedbacks in a patchy environment was investigated in reference [44]. They obtained global stability criteria of the disease-free and endemic states. The authors determined the global 
stability of endemic state using some results of graph theory. The global stability of epidemic models with feedback and the effects of feedback on the transmission dynamics of disease have been investigated in [45-47]. Thus, we rarely find few studies on the effect of feedback in multi-group epidemic models incorporating the effect of age of infection. Moreover, the impact of the infection age on the susceptible individuals has not been addressed in the presence of feedback variables. However, the study of the multi-group epidemic model with infection age and feedback can significantly contribute to the control of infection in more realistic situations. Motivated from above cited works, we propose a multi-group SEIR epidemic model system with infectivity as a function of infection age and feedback with the following objectives:

1. To establish the global dynamics of the model system using graph theoretic results.

2. To improve the understanding how feedback and age of infection influence the transmission dynamics of infectious diseases.

Graph-Theoretic Approach to Multi-group Epidemic Model: To understand the dynamics of model, basic reproduction number $\left(R_{0}\right)$ plays an important role. One tries to the study the case $R_{0} \leq 1$ and $R_{0}>1$. For the case $R_{0} \leq 1$, there exists only one (disease free) equilibria and for the case $R_{0}>1$, there exist at least two equilibrium, one is called disease free equilibrium and the other one is known as endemic equilibrium. To study the global stability of an equilibrium, one tries to construct a suitable Lyapunov function. In general, the construction of a Lyapunov function is a difficult problem. In references [48-49], the authors elaborated a graph theoretic technique to study the global dynamics of endemic equilibrium by constructing a Lyapunov function. The method involves the complete description of the complicated pattern to construct a Lyapunov function. There are a few multi-group epidemic models (see [50-54]), in which this graph theoretic approach is used to determine the global stability of endemic equilibria of models. This method has been used to determine the global stability of unique endemic equilibrium of a multi-group SIR epidemic model which is described by ordinary differential equations (see [48]).

For basic notions of the graph theory, we refer the interested readers to [55-56]. Now, we mention some results to be used in the present paper.

Definition 1.1 (see [55]) Let $B=\left(\beta_{k j}\right)_{n \times n}$ be a real matrix. If $\beta_{k j}$ are nonnegative for all $k$ and $j$, then $B$ is called non-negative matrix (i.e., $B \geq 0$ ). If $B$ and a $F=\left(f_{k j}\right)_{n \times n}$ are both non-negative, then $B-F \geq 0$ if and only if $\beta_{k j} \geq f_{k j}$ for all $k$ and $j$.

Definition 1.2 (see [55]) Let $B=\left(\beta_{k j}\right)_{n \times n}$ be a non-negative matrix. If $B$ satisfies one of the following properties, then $B$ is called reducible

1. $n=1$ and $B=0$,

2. $n \geq 2$, there exits a permutation matrix $P$, such that

$$
P B P^{\mathrm{T}}=\left(\begin{array}{cc}
B_{1} & 0 \\
B_{2} & B_{3}
\end{array}\right)
$$

where $B_{1}$ and $B_{2}$ are square matrices and $P^{\mathrm{T}}$ is the transpose of matrix $P$. Otherwise, $B$ is called irreducible. 
We consider the linear system

$$
\bar{B} v=0
$$

where

$$
\bar{B}=\left[\begin{array}{cccc}
\sum_{l \neq 1} \bar{\beta}_{1 l} & -\bar{\beta}_{21} & \cdots & -\bar{\beta}_{n 1} \\
\bar{\beta}_{12} & \sum_{l \neq 2} \bar{\beta}_{1 l} & \cdots & -\bar{\beta}_{n 2} \\
\vdots & \vdots & & \vdots \\
-\bar{\beta}_{1 n} & -\bar{\beta}_{n 2} & \cdots & \sum_{l \neq n} \bar{\beta}_{n l}
\end{array}\right], \quad \bar{\beta}_{k j} \geq 0
$$

is the Laplacian matrix of the directed graph $G(B)$ associated to the matrix $B$.

Lemma 1.1 (see [57]) If $B$ is non-negative and irreducible, then

(1) the spectral radius $\rho(B)$ of $B$ is a simple eigenvalue of $B$, and $B$ has a positive eigenvector $c=\left(c_{1}, c_{2}, \cdots, c_{n}\right)$ corresponding to $\rho(B)$.

(2) If $B \leq F$, then $\rho(B) \leq \rho(F)$ and furthermore, if $B<F$ and $B+F$ is irreducible, then $\rho(B)<\rho(F)$.

(3) If $F$ is a diagonal and positive (i.e., all entries of $F$ are positive) matrix, then BF is irreducible.

Lemma 1.2 (see [48]) If the matrix $\bar{B}$ is irreducible and $n \geq 2$, then the following properties hold.

(1) The solution of linear system (1.1) is the space of dimension 1 , with a basis $\left(v_{1}, v_{2}, \cdots\right.$, $\left.v_{n}\right)=\left(K_{11}, K_{22}, \cdots, K_{n n}\right)$, where $K_{i i}$ is the cofactor of the $i$-th diagonal entry of matrix $\bar{B}$, $1 \leq i \leq n$.

(2) For all $1 \leq i \leq n$,

$$
K_{i i}=\sum_{T \in \mathbb{T}_{i}} \prod_{(k, j) \in E(T)} \bar{\beta}_{k j} \quad \text { and } \quad K_{i i}>0
$$

where $\mathbb{T}_{i}$ is the set of all spanning subtrees of vertices of $G(B)$ that are rooted at vertex $i$ and $E(T)$ is the set of all arcs of directed tree $T$.

The rest of the paper is organized as follows. In Section 2, we mathematically formulate our problem considering some basic assumptions. In Section 3, we prove the well-posedness (positivity and boundedness) of the proposed model system. In Section 4, we prove our main results about the global asymptotic stability of the disease free equilibrium and the endemic equilibrium of model system. In Section 5, numerical evaluations have been presented to support our theoretical results by taking an example of 2 -group populations. Finally, in Section 6 , we discuss our results including some ideas about future scope.

\section{Mathematical Model Formulation}


The heterogeneous host population is divided into $n$ homogeneous groups of population according to gender, age, profession, education levels and geographical distribution for their heterogeneity to disease transmission. Further, we divide any $k$-th group into four compartments $\left(S_{k}, E_{k}, I_{k}, R_{k}\right)$ to study our problem as compartmental model of epidemic, where $1 \leq k \leq n$. Let $S_{k}$ be the susceptible individuals who are at risk of infection of disease, $E_{k}$ be the individuals of exposed class who are infected by disease but do not liable to spread disease, the infectious individuals $I_{k}$ who are infected by disease and they will influence susceptible individuals, the recovered individuals $R_{k}$ who are the recovered and have permanent immunity against the disease. We also assume that the susceptible individuals $S_{k}$, the exposed individuals $E_{k}$ and the recovered individuals are homogeneous at any time $t$ in the $k$-th group and the infectious individuals $I_{k}$ is structured by the infection age $\theta$ and $i_{k}(t, \theta)$ is the density of infectious individuals with infection age $\theta$ at the time $t$ in $k$-th group. We assume that $i_{k}(t, \theta)=0$ for all $\theta>\theta^{*}$ (finite), where $\theta^{*}$ is the maximum infection age, for which the infectious individual can survive, that means an individual can stay in infectious class for $\theta^{*}$ unit time. Then $I_{k}(t)=\int_{0}^{\infty} i_{k}(t, \theta)$ is the total number of infectious individuals at time $t$. We make the following assumptions for our model system:

(A1) In $k$-th group, new recruits in the total population take place at a rate $\Gamma_{k}>0$ at any moment of time. In which $\left(1-p_{k}\right)$ proportion of new recruits enter in the susceptible population and remaining $p_{k}$ proportion enter in the recovered population. That means some proportion of new recruiting population could not be susceptible to infection due to having permanent immunity.

(A2) In $k$-th group, by using the vaccine against the virus/bacteria and provided immunity against the various diseases, the susceptible individuals $\left(S_{k}\right)$ enter to recovered class $\left(R_{k}\right)$ at a constant rate $\delta_{k}>0$.

(A3) For $k$-th group, $U_{k}$ is feedback variable which satisfies certain differential equation and it influences susceptible individuals by a rate $b_{k} U_{k}$, where $b_{k}$ is the feedback parameter.

(A4) In $k$-th group, after the latent period, the individuals of exposed class turn into infectious individual class with a constant rate $\varepsilon_{k}>0$.

(A5) The infected individuals in $I_{k}$ class can recover (through treatment or automatically) and get permanent immunity against disease, i.e., $\gamma_{k}$ is the recovery rate of $I_{k}$.

(A6) Here, we consider the cross-infection from all groups of infectious individuals to a group of susceptible individuals. Let $h_{k}(\theta)$ be a bounded and non-negative continuous function of $\theta$, which represents the infectivity of infected individuals of $\theta$ infection age of $k$-th group.

(A7) In $k$-th group, the coefficient of infection transmission for susceptible individuals $S_{k}$ turning into exposed individuals $E_{k}$ is $\beta_{k j} \geq 0$, in which a susceptible individual makes contact with infectious individuals $I_{k}$ of the $j$-th group. For any two distinct groups ( $k$-th and $j$-th), individuals of $I_{j}$ can infect individuals of $S_{k}$ in direct or indirect mode, i.e., $\beta_{k j}$ is irreducible matrix.

Under the above assumptions and discussions, the proposed multi-group SEIR model system 
is

$$
\begin{aligned}
& \frac{\mathrm{d} S_{k}}{\mathrm{~d} t}=\left(1-p_{k}\right) \Gamma_{k}-\left(d_{k}^{S}+\delta_{k}\right) S_{k}-\sum_{j=1}^{n} \beta_{k j} S_{k} \int_{0}^{\infty} h_{j}(\theta) i_{j}(t, \theta) \mathrm{d} \theta-b_{k} U_{k} S_{k}, \\
& \frac{E_{k}}{\mathrm{~d} t}=\sum_{j=1}^{n} \beta_{k j} S_{k} \int_{0}^{\infty} h_{j}(\theta) i_{j}(t, \theta) \mathrm{d} \theta-\left(d_{k}^{E}+\varepsilon_{k}\right) E_{k}, \\
& \left(\frac{\partial}{\partial t}+\frac{\partial}{\partial \theta}\right) i_{k}(t, \theta)=-\left(d_{k}^{I}+\gamma_{k}\right) i_{k}(t, \theta) \\
& \frac{\mathrm{d} U_{k}}{\mathrm{~d} t}=-f_{k} U_{k}+e_{k} S_{k} \\
& \frac{\mathrm{d} R_{k}}{\mathrm{~d} t}=p_{k} \Gamma_{k}+\delta_{k} S_{k}+\gamma_{k} \int_{0}^{\infty} i_{k}(t, \theta) \mathrm{d} \theta-d_{k}^{R} R_{k}
\end{aligned}
$$

where $k=1,2, \cdots, n$ and $U_{k}$ is the $k$-th feedback variable.

The initial and boundary conditions of model system (2.1) are given by:

$$
\begin{aligned}
S_{k}(0) & =\phi_{1}^{k} \geq 0, \quad E_{k}(0)=\phi_{2}^{k} \geq 0, \quad R_{k}(0)=\phi_{3}^{k} \geq 0, \quad U_{k}(0)=\phi_{4}^{k} \geq 0, \\
i_{k}(0, \theta) & =\phi_{k}^{0}(\theta) \in L_{+}(0, \infty) \quad \text { and } \quad i_{k}(t, 0)=\varepsilon_{k} E_{k}(t),
\end{aligned}
$$

where $L_{+}(0, \infty)$ is the space of the non-negative functions.

In an age-structured infection age model, the variable $i_{k}(t, \theta)$ has two interpretations. Firstly, it can be interpreted as the density of infected individuals of infection age $\theta$ at time $t$ (this means the actual number of infected individuals at time $t$ between two infection ages $\theta_{1}$ and $\theta_{2}$ will be the integral of the density function $i_{k}(t, \theta)$ over $\theta \in\left(\theta_{1}, \theta_{2}\right)$. Secondly, the density function $i_{k}(t, \theta)$ evaluated at $(t, a)$ has the interpretation of being the rate at time $t$ at which individuals pass through age $a$. As a consequence, $i_{h}(t, 0)$ is the overall birth rate for infected individuals (rate at which the exposed individuals become infected).

The total number of infected individuals is found by integrating the density $i_{k}(t, \theta)$ over all ages $\theta \in[0, \infty)$ as follows:

$$
I_{k}=\int_{0}^{\infty} i_{k}(t, \theta) \mathrm{d} \theta
$$

Note that we assume that the disease confers permanent immunity in the above model. This assumption makes the first four equations of model system (2.1) independent from $R_{k}$. Therefore, the dynamics of our model is governed by the following reduced system:

$$
\begin{aligned}
& \frac{\mathrm{d} S_{k}}{\mathrm{~d} t}=\left(1-p_{k}\right) \Gamma_{k}-\left(d_{k}^{S}+\delta_{k}\right) S_{k}-\sum_{j=1}^{n} \beta_{k j} S_{k} \int_{0}^{\infty} h_{j}(\theta) i_{j}(t, \theta) \mathrm{d} \theta-b_{k} U_{k} S_{k} \\
& \frac{\mathrm{d} E_{k}}{\mathrm{~d} t}=\sum_{j=1}^{n} \beta_{k j} S_{k} \int_{0}^{\infty} h_{j}(\theta) i_{j}(t, \theta) \mathrm{d} \theta-\left(d_{k}^{E}+\varepsilon_{k}\right) E_{k} \\
& \left(\frac{\partial}{\partial t}+\frac{\partial}{\partial \theta}\right) i_{k}(t, \theta)=-\left(d_{k}^{I}+\gamma_{k}\right) i_{k}(t, \theta) \\
& \frac{\mathrm{d} U_{k}}{\mathrm{~d} t}=-f_{k} U_{k}+e_{k} S_{k} .
\end{aligned}
$$


Now, let $\psi(\theta)=\mathrm{e}^{-\int_{0}^{\theta}\left(d_{j}^{I}+\gamma_{j}\right) \mathrm{d} a}$. Then $\psi(\theta)$ is the probability of an infected individual in the $j$-th group surviving to infection age $\theta$. Integrating the third equation of model system (2.1) and incorporating the initial conditions, we obtain

$$
i_{k}(t, \theta)=\varepsilon_{k} E_{k}(t-\theta) \mathrm{e}^{-\left(d_{k}^{I}+\gamma_{k}\right) \theta} .
$$

Let $f_{j}(\theta)=h_{j}(\theta) \varepsilon_{j} \mathrm{e}^{-\left(d_{j}^{I}+\gamma_{j}\right) \theta}$ be the general kernel function. Then by (2.3) and (2.4), we obtain the following model system:

$$
\begin{aligned}
\frac{\mathrm{d} S_{k}}{\mathrm{~d} t} & =\left(1-p_{k}\right) \Gamma_{k}-\left(d_{k}^{S}+\delta_{k}\right) S_{k}-\sum_{j=1}^{n} \beta_{k j} S_{k} \int_{0}^{\infty} f_{j}(\theta) E_{j}(t-\theta) \mathrm{d} \theta-b_{k} U_{k} S_{k}, \\
\frac{\mathrm{d} E_{k}}{\mathrm{~d} t} & =\sum_{j=1}^{n} \beta_{k j} S_{k} \int_{0}^{\infty} f_{j}(\theta) E_{j}(t-\theta) \mathrm{d} \theta-\left(d_{k}^{E}+\varepsilon_{k}\right) E_{k}, \\
\frac{\mathrm{d} U_{k}}{\mathrm{~d} t} & =-f_{k} U_{k}+e_{k} S_{k} .
\end{aligned}
$$

Here the kernel function $f_{k}(\theta)(\geq 0)$ is a continuous function of the infection age $\theta$ with $\int_{\theta=0}^{\infty} f_{k}(\theta) \mathrm{d} \theta=a_{k}>0$. Our assumption on the kernel function $f_{k}(\theta)$ is $\int_{\theta=0}^{\infty} f_{k}(\theta) \mathrm{e}^{\lambda_{k} \theta} \mathrm{d} \theta<\infty$, where $\lambda_{k}$ is a positive number, $k=1,2, \cdots, n$. System (2.5) can be interpreted as a multigroup (say $n$-group) epidemic model for an infectious disease whose latent period $\theta$ in the host is obtained from the general age of infection model (2.1). Here, we also realize that the model system (2.5) is an epidemic model with distributed time delay.

If we take $\int_{0}^{\infty} i_{j}(t, \theta) \mathrm{d} \theta=I_{j}$ and $h_{j}(\theta)=1$, then model system (2.1) changes into associated ODE system of the following form:

$$
\begin{aligned}
\frac{\mathrm{d} S_{k}}{\mathrm{~d} t} & =\left(1-p_{k}\right) \Gamma_{k}-\left(d_{k}^{S}+\delta_{k}\right) S_{k}-\sum_{j=1}^{n} \beta_{k j} S_{k} I_{j}-b_{k} U_{k} S_{k} \\
\frac{\mathrm{d} E_{k}}{\mathrm{~d} t} & =\sum_{j=1}^{n} \beta_{k j} S_{k} I_{j}-\left(d_{k}^{E}+\varepsilon_{k}\right) E_{k} \\
\frac{\mathrm{d} I_{k}}{\mathrm{~d} t} & =\varepsilon_{k} E_{k}-\left(d_{k}^{I}+\gamma_{k}\right) I_{k} \\
\frac{\mathrm{d} U_{k}}{\mathrm{~d} t} & =-f_{k} U_{k}+e_{k} S_{k} \\
\frac{\mathrm{d} R_{k}}{\mathrm{~d} t} & =p_{k} \Gamma_{k}+\delta_{k} S_{k}+\gamma_{k} I_{k}-d_{k}^{R} R_{k}
\end{aligned}
$$

The dynamical behaviour of model system (2.3) is equivalent to that of system (2.5). Once we determine the solution of system $(2.5)$, we can calculate $i_{k}(t, \theta)$ from (2.4). So, the stability of equilibria of model system (2.1) is the same as that of system (2.5). From here onward, we shall focus on the model system $(2.5)$.

\section{The Well-Posedness of Model}

Due to infinite delay, it is necessary to determine the suitable phase space of state varibales. Therefore, we define the following Banach space of fading memory type (see [58]) for any 
$\lambda_{k} \in\left(0, d_{k}^{I}+\gamma_{k}\right)$

$$
\begin{gathered}
C_{k}=\left\{\Psi \in C((-\infty, 0], \mathbb{R}) \mid \Psi(r) \mathrm{e}^{\lambda_{k} r}\right. \text { is uniformly continuous on } \\
\left.(0,-\infty] \text { with } \sup _{r \leq 0}|\Psi(r)| \mathrm{e}^{\lambda_{k} r}<\infty\right\}
\end{gathered}
$$

and norm defined by $\|\Psi\|_{k}=\sup _{r \leq 0}|\Psi(r)| \mathrm{e}^{\lambda_{k} r}$. Now for $\Psi \in C_{k}$, let $\Psi_{t} \in C_{k}$ be defined by $\Psi_{t}(r)=$ $\Psi(t+r), r \in(-\infty, 0]$. Let $S_{k, 0}, U_{k, 0} \in \mathbb{R}_{+}$and $\Psi_{k} \in C_{k}$ such that $\Psi_{k}(r) \geq 0, r \in(-\infty, 0]$. For our case $\Psi=E_{k}$. Thus, $E_{k}(t-\theta)=E_{k t}(\theta), \theta \in[0, \infty)$. Now we consider the solution $\left(S_{1}(t), E_{1 t}, U_{1}(t), S_{2}(t), E_{2 t}, U_{2}(t), \cdots, S_{n}(t), E_{n t}, U_{n}(t)\right)$ of model system (2.5) with the initial conditions

$$
S_{k}(0)=S_{k, 0}, \quad E_{k 0}=\Psi_{k}, \quad U_{k}(0)=U_{k, 0}, \quad k=1,2, \cdots, n .
$$

Then, by standard theory of functional differential equations (see [59]), we have $E_{k t} \in C_{k}$. Therefore, we consider model system (2.5) in the phase space

$$
\Delta=\prod_{k=1}^{n}\left(\mathbb{R} \times C_{k} \times \mathbb{R}\right)
$$

Considering the biological meaning, we are only interested in the solutions which are nonnegative and bounded. Now, we show that all the solutions of model system (2.5) with initial conditions (3.1) are non-negative, i.e., $S_{k}(t) \geq 0, E_{k}(t) \geq 0, U_{k}(t) \geq 0$ for all $t \geq 0$ and $k \in\{1,2, \cdots, n\}$. Let $S_{k}(t)>0$ with initial value $S_{k}(0)>0$ for all $k \in\{1,2, \cdots, n\}$. We suppose that it is not correct, then there exist a positive number $t_{1}$ and some $k_{1} \in\{1,2, \cdots, n\}$ such that

$$
S_{k_{1}}(t)>0, \quad S_{k_{1}}\left(t_{1}\right)=0, \quad 0 \leq t<t_{1} .
$$

It follows from the first equation of model system (2.5)

$$
\dot{S}_{k_{1}}(t)>S_{k_{1}}\left(-\left(d_{k_{1}}^{s}+\delta_{k_{1}}\right)-\sum_{j=1}^{n} \beta_{k_{1} j} \int_{0}^{\infty} f_{j}(\theta) E_{j}(t-\theta) \mathrm{d} \theta-b_{k_{1}} U_{k_{1}}\right), \quad 0 \leq t<t_{1} .
$$

Using the comparison theorem (see [60]) and taking the limit as $t \rightarrow t_{1}$, we obtain

$$
S_{k_{1}}\left(t_{1}\right)>S_{k_{1}}(0) \exp \int_{0}^{t_{1}}\left(-\left(d_{k_{1}}^{s}+\delta_{k_{1}}\right)-\sum_{j=1}^{n} \beta_{k_{1} j} \int_{0}^{\infty} f_{j}(\theta) E_{j}(r-\theta) \mathrm{d} \theta-b_{k_{1}} U_{k_{1}}(r)\right) \mathrm{d} r>0,
$$

which contradicts our supposition that $S_{k_{1}}\left(t_{1}\right)=0$. Therefore, we conclude that if $S_{k}(0)>0$ then $S_{k}(t)>0$ for all $t \geq 0$ and $k \in\{1,2, \cdots, n\}$. From the continuity of solution of system (2.5) around the initial condition, we have that if $S_{k}(0) \geq 0$, then $S_{k}(t) \geq 0$ for all $t \geq 0$ and $k \in\{1,2, \cdots, n\}$.

Similarly, let $E_{k}(t)>0$ with initial value $E_{k}(0)>0$ for all $k \in\{1,2, \cdots, n\}$. We assume that it is not correct and there exist a positive number $t_{2}$ and $k_{2} \in\{1,2, \cdots, n\}$ such that

$$
E_{k_{2}}(t)>0, \quad E_{k_{2}}\left(t_{2}\right)=0, \quad 0 \leq t<t_{2} .
$$


, here, $0<t_{2}-\theta<t_{2}$. Hence, we have $E_{k_{2}}\left(t_{2}-\theta\right)>0$. Then from the second equation of model system (2.5), we obtain $\frac{\mathrm{d} E_{k_{2}}\left(t_{2}\right)}{\mathrm{d} t}>0$ and $E_{k_{2}}(t)>0$ for all $t>t_{2}$. This is a contradiction to our assumption. Therefore, from continuity of solution of system (2.5) around the initial value, we conclude that $E_{k}(0) \geq 0$. Thus $E_{k}(t) \geq 0$ for all $t \geq 0$ and $k \in\{1,2, \cdots, n\}$, we have

Now, from the last equation of system (2.5):

$$
\frac{\mathrm{d} U_{k}(t)}{\mathrm{d} t} \geq-f_{k} U_{k}(t) \quad \text { for all } k \in\{1,2, \cdots, n\} .
$$

By the comparison theorem (see [60]), we obtain

$$
U_{k}(t) \geq U_{k}(0) \exp \left(-f_{k} t\right)>0 \quad \text { for all } k \in\{1,2, \cdots, n\}
$$

Thus, we conclude that if $U_{k}(0) \geq 0$, then $U_{k}(t) \geq 0$ for all $t \geq 0$ and $k \in\{1,2, \cdots, n\}$.

Now, we show that solutions of system (2.5) with initial and boundary conditions (3.1) are bounded. For this, we have $S_{k}(t)>0$ for $t>0$. Thus, from the first equation of system (2.5), we obtain $S_{k}^{\prime}(t) \leq\left(1-p_{k}\right) \Gamma_{k}-\left(d_{k}^{s}+\delta_{k}\right) S_{k}(t)$. Hence,

$$
\lim \sup _{t \rightarrow \infty} S_{k}(t) \leq \frac{\left(1-p_{k}\right) \Gamma_{k}}{d_{k}^{s}+\delta} .
$$

By adding all the equations of system (2.5) for each $k$, we obtain

$$
\begin{aligned}
\left(S_{k}(t)+E_{k t}(0)+U_{k}(t)\right)^{\prime} & =\left(1-p_{k}\right) \Gamma_{k}-\left(d_{k}^{s}+\delta_{k}\right) S_{k}-b_{k} U_{k} S_{k}-\left(d_{k}^{E}+\varepsilon_{k}\right) E_{k}-f_{k} U_{k}+e_{k} S_{k} \\
& \leq\left(1-p_{k}\right) \Gamma_{k}-d_{k}^{*}\left(S_{k}(t)+E_{k t}(0)+U_{k}(t)\right)
\end{aligned}
$$

where $d_{k}^{*}=\min \left\{\left(d_{k}^{s}+\delta_{k}-e_{k}\right),\left(d_{k}^{E}+\varepsilon_{k}\right), f_{k}\right\}$ and $\left(d_{k}^{s}+\delta_{k}-e_{k}\right)>0$.

Thus, we obtain

$$
\lim \sup _{t \rightarrow \infty}\left(S_{k}(t)+E_{k t}(0)+U_{k}(t)\right) \leq \frac{\left(1-p_{k}\right) \Gamma_{k}}{d_{k}^{*}} .
$$

Therefore, the following region is positively invariant for system (2.5)

$$
\begin{aligned}
& \xi=\left\{\left(S_{1}, E_{1}(\cdot), U_{1}, \cdots, S_{n}, E_{n}(\cdot), U_{n}\right) \in \Delta \mid 0 \leq S_{k} \leq \frac{\left(1-p_{k}\right) \Gamma_{k}}{d_{k}^{s}+\delta},\right. \\
& \left.0 \leq\left(S_{k}(t)+E_{k t}(0)+U_{k}(t)\right) \leq \frac{\left(1-p_{k}\right) \Gamma_{k}}{d_{k}^{*}}, E_{k}(r) \geq 0, r \in(-\infty, 0]\right\},
\end{aligned}
$$

and

$$
\begin{aligned}
& \bar{\xi}=\left\{\left(S_{1}, E_{1}(\cdot), U_{1}, \cdots, S_{n}, E_{n}(\cdot), U_{n}\right) \in \Delta \mid 0<S_{k}<\frac{\left(1-p_{k}\right) \Gamma_{k}}{d_{k}^{s}+\delta},\right. \\
& \left.0<\left(S_{k}(t)+E_{k t}(0)+U_{k}(t)\right)<\frac{\left(1-p_{k}\right) \Gamma_{k}}{d_{k}^{*}}, E_{k}(r) \geq 0, r \in(-\infty, 0]\right\}
\end{aligned}
$$

is interior set of $\xi$. Therefore $\bar{\xi}$ is also positively invariant for system (2.5). 


\section{Equilibria and Their Global Stability}

System (2.5) always has a disease free equilibrium $P_{0}=\left(S_{1}^{0}, 0, U_{1}^{0}, S_{2}^{0}, 0, U_{2}^{0}, \cdots, S_{n}^{0}, 0, U_{n}^{0}\right)$ $\in \mathbb{R}_{+}^{3 n}$, where

$$
S_{k}^{0}=\frac{-f_{k}\left(d_{k}^{S}+\delta_{k}\right)+\sqrt{\left(f_{k}\left(d_{k}^{S}+\delta_{k}\right)\right)^{2}+4 b_{k} e_{k}\left(1-p_{k}\right) \Gamma_{k} f_{k}}}{2 b_{k} e_{k}}, \quad U_{k}^{0}=\frac{e_{k} S_{k}^{0}}{f_{k}}
$$

The endemic equilibrium of system (2.5) is given by

$$
P^{*}=\left(S_{1}^{*}, E_{1}^{*}, U_{1}^{*}, S_{2}^{*}, E_{2}^{*}, U_{2}^{*} \cdots, S_{n}^{*}, E_{n}^{*}, U_{n}^{*}\right) \in \bar{\Xi}
$$

and it is calculated by solving the following system of equations

$$
\begin{aligned}
0 & =\left(1-p_{k}\right) \Gamma_{k}-\left(d_{k}^{S}+\delta_{k}\right) S_{k}^{*}-\sum_{j=1}^{n} \beta_{k j} a_{j} S_{k}^{*} E_{j}^{*}-b_{k} U_{k}^{*} S_{k}^{*}, \\
0 & =\sum_{j=1}^{n} \beta_{k j} a_{j} S_{k}^{*} E_{j}^{*}-\left(d_{k}^{E}+\varepsilon_{k}\right) E_{k}^{*}, \\
0 & =-f_{k} U_{k}^{*}+e_{k} S_{k}^{*},
\end{aligned}
$$

where $a_{j}=\int_{0}^{\infty} f_{j}(\theta) \mathrm{d} \theta$. In epidemiology, the basic reproduction number $R_{0}$ is defined as the total expected number of secondary cases produced by an infected individual during its total infectious period, in an entirely susceptible population (see [61]). The basic reproduction number $R_{0}$ is determined by the spectral radius of a matrix $Q_{0}$ defined as $Q_{0}=q_{k j}$, where $q_{k j}=\left(\frac{\beta_{k j} S_{k}^{0} a_{j}}{d_{k}^{E}+\varepsilon_{k}}\right)_{n \times n}$.

Therefore

$$
R_{0}=\rho\left(Q_{0}\right)
$$

where $\rho\left(Q_{0}\right)$ denotes the spectral radius of matrix $Q_{0}$. In epidemiology, $R_{0}$ plays a major role to determine the dynamical behavior of system and it acts as threshold. We shall determine the dynamical behavior of system (2.5) completely in terms of $R_{0}$.

\subsection{Global stability of disease free equilibrium (DFE for short)}

Let $S=\left(S_{1}, S_{2}, \cdots, S_{n}\right)$ and $S^{0}=\left(S_{1}^{0}, S_{2}^{0}, \cdots, S_{n}^{0}\right)$. Then $Q_{0}=Q\left(S^{0}\right)$. Since $0 \leq S_{k} \leq S_{k}^{0}$ for all $k$, hence, we have $0 \leq Q(S) \leq Q\left(S^{0}\right)=M_{0}$. If $S \neq S^{0}$, then $Q(S)<Q_{0}$. Since $B=\beta_{k j}$ is an irreducible matrix, $Q(S)$ and $Q\left(S^{0}\right)$ are irreducible matrices. Then by Lemma 1.1, $Q(S)$ $+Q\left(S^{0}\right)$ is also an irreducible matrix and $\rho(Q(S))<\rho\left(Q_{0}\right)$ provided $S \neq S^{0}$. Now we prove the following theorem.

Theorem 4.1 Assume that $B=\left(\beta_{k j}\right)_{n \times n}$ is irreducible. If $R_{0} \leq 1$, then DFE of system (2.5) is globally asymptotically stable. Moreover, if $R_{0}>1$, then DFE is unstable.

Proof We know that matrix $Q=Q_{0}=\left(\frac{\beta_{k j} S_{k}^{0} a_{j}}{d_{k}^{E}+\varepsilon_{k}}\right)_{n \times n}$ is irreducible. Therefore, by Lemma 1.1 , the matrix $Q$ has a positive left eigenvector $\left(w_{1}, w_{2}, \cdots, w_{n}\right)$ corresponding to the spectral 
radius of matrix $(\rho(Q)>0)$. In particular, $\rho(Q)=\rho\left(Q_{0}\right)=R_{0} \leq 1$. Let $c_{k}=\frac{w_{k}}{d_{k}^{E}+\varepsilon_{k}}>0$ and $a_{k}(\theta)=\int_{\sigma=\theta}^{\infty} f_{k}(\sigma) \mathrm{d} \sigma$. Thus, $a_{k}(0)=\int_{\sigma=0}^{\infty} f_{k}(\sigma) \mathrm{d} \sigma=a_{k}$.

Now, we consider the following Lyapunov function:

$$
\begin{aligned}
V= & \sum_{k=1}^{n} c_{k}\left(S_{k}-S_{k}^{0}-S_{k}^{0} \log \frac{S_{k}}{S_{k}^{0}}+E_{k}\right. \\
& \left.+\sum_{j=1}^{n} \beta_{k j} S_{k}^{0} \int_{0}^{\infty} a_{j}(\theta) E_{j}(t-\theta) \mathrm{d} \theta+\frac{b_{k}}{2 e_{k}}\left(U_{k}-U_{k}^{0}\right)^{2}\right) .
\end{aligned}
$$

Note that $\frac{S_{k}}{S_{k}^{0}}+\frac{S_{k}^{0}}{S_{k}} \geq 2$ and equality holds if and only if $S_{k}=S_{k}^{0}$. Now, by differentiating $V$ with respect to solutions of model system (2.5), we obtain

$$
\begin{aligned}
V^{\prime}= & \sum_{k=1}^{n} c_{k}\left[\left(1-\frac{S_{k}^{0}}{S_{k}}\right) S_{k}^{\prime}+E_{k}^{\prime}+\sum_{j=1}^{n} \beta_{k j} S_{k}^{0} \int_{0}^{\infty} a_{j}(\theta) \frac{\partial E_{j}(t-\theta)}{\partial t} \mathrm{~d} \theta+\frac{b_{k}}{e_{k}}\left(U_{k}-U_{k}^{0}\right) U_{k}^{\prime}\right] \\
= & \sum_{k=1}^{n} c_{k}\left(\left(1-p_{k}\right) \Gamma_{k}-\left(d_{k}^{s}+\delta_{k}\right) S_{k}-b_{k} U_{k} S_{k}-\left(1-p_{k}\right) \Gamma_{k} \frac{S_{k}^{0}}{S_{k}}\right. \\
& +\sum_{j=1}^{n} \beta_{k j} S_{k}^{0} \int_{0}^{\infty} f_{j}(\theta) E_{j}(t-\theta) \mathrm{d} \theta+\left(d_{k}^{s}+\delta_{k}\right) S_{k}^{0}+b_{k} U_{k} S_{k}^{0} \\
& -\left(d_{k}^{E}+\varepsilon_{k}\right) E_{k}+\sum_{j=1}^{n} \beta_{k j} S_{k}^{0} \int_{0}^{\infty} a_{j}(\theta) \frac{\partial E_{j}(t-\theta)}{\partial t} \mathrm{~d} \theta \\
& \left.+\frac{b_{k}}{e_{k}}\left(U_{k}-U_{k}^{0}\right)\left(e_{k} S_{k}-f_{k} U_{k}\right)\right) .
\end{aligned}
$$

Now, by using the disease free stationary state and integration by parts, we obtain

$$
\begin{aligned}
V^{\prime}= & \sum_{k=1}^{n} c_{k}\left(d_{k}^{s}+\delta_{k}+b_{k} U_{k}^{0} S_{K}^{0}\right)\left(2-\frac{S_{k}}{S_{k}^{0}}-\frac{S_{k}^{0}}{S_{k}}\right)-\frac{b_{k}}{e_{k}} f_{k}\left(U_{k}-U_{k}^{0}\right)^{2} \\
& +\sum_{j=1}^{n} \beta_{k j} S_{k}^{0} \int_{0}^{\infty} f_{j}(\theta) E_{j}(t-\theta) \mathrm{d} \theta-\left(d_{k}^{0}+\varepsilon_{k}\right) E_{k}+\sum_{j=1}^{n} \beta_{k j} S_{k}^{0} \int_{0}^{\infty} a_{j}(\theta) \frac{\partial E_{j}(t-\theta)}{\partial t} \mathrm{~d} \theta \\
\leq & \sum_{k=1}^{n} c_{k}\left[\sum_{j=1}^{n} \beta_{k j} S_{k}^{0} \int_{0}^{\infty} f_{j}(\theta) E_{j}(t-\theta) \mathrm{d} \theta-\left(d_{k}^{0}+\varepsilon_{k}\right) E_{k}+\sum_{j=1}^{n} \beta_{k j} S_{k}^{0} \int_{0}^{\infty} a_{j}(\theta) \frac{\partial E_{j}(t-\theta)}{\partial t} \mathrm{~d} \theta\right] \\
= & \sum_{k=1}^{n} c_{k}\left[\sum_{j=1}^{n} \beta_{k j} S_{k}^{0} \int_{0}^{\infty} f_{j}(\theta) E_{j}(t-\theta) \mathrm{d} \theta-\left(d_{k}^{0}+\varepsilon_{k}\right) E_{k}\right. \\
& \left.+\sum_{j=1}^{n} \beta_{k j} S_{k}^{0}\left(a_{j} E_{j}-\int_{0}^{\infty} f_{j}(\theta) E_{j}(t-\theta) \mathrm{d} \theta\right)\right] \\
= & \sum_{k=1}^{n} w_{k}\left[\sum_{j=1}^{n} \frac{\beta_{k j} a_{j} S_{k}^{0} E_{j}}{d_{k}^{E}+\varepsilon_{k}}-E_{k}\right]=\left(w_{1}, w_{2}, \cdots, w_{k}\right)(Q E-E) \\
= & \left(\rho\left(Q_{0}\right)-1\right)\left(w_{1}, w_{2}, \cdots, w_{k}\right) E=\left(R_{0}-1\right)\left(w_{1}, w_{2}, \cdots, w_{k}\right) E \\
\leq & 0, \quad \text { if } R_{0} \leq 1,
\end{aligned}
$$

where $E(t)=\left(E_{1}(t), E_{2}(t), \cdots, E_{n}(t)\right)^{\mathrm{T}}$. 
Let $Y=\left\{\left(S_{1}, E_{1}(\cdot), U_{1}, \cdots, S_{n}, E_{n}(\cdot), U_{1}\right) \in \xi \mid V^{\prime}=0\right\}$ and $Z$ be the largest compact invariant subset of $Y$. Now, we prove that $Z=\left\{P_{0}\right\}$. From inequality (4.3) and assumption $c_{k}>0$, if $L^{\prime}=0$ then $\left(2-\frac{S_{k}}{S_{k}^{0}}-\frac{S_{k}^{0}}{S_{k}}\right)=0$ and $\left(U_{k}-U_{k}^{0}\right)^{2}=0$. Therefore, $S_{k}(t)=S_{k}^{0} \neq 0$ and $U_{k}=U_{k}^{0} \neq 0$. Hence, from the first equation of system (2.5), we have

$$
\sum_{j=1}^{n} \beta_{k j} \int_{0}^{\infty} f_{j}(\theta) E_{j}(t-\theta) \mathrm{d} \theta=0,
$$

and thus

$$
\beta_{k j} \int_{0}^{\infty} f_{j}(\theta) E_{j}(t-\theta) \mathrm{d} \theta=0 \quad \text { for all } 1 \leq k, j \leq n .
$$

Note that $B=\left(\beta_{k j}\right)_{n \times n}$ is irreducible. So, for each $1 \leq j \leq n$ and $k \neq j$, we have $\beta_{k j} \neq 0$. Therefore, we obtain

$$
\int_{0}^{\infty} f_{j}(\theta) E_{j}(t-\theta) \mathrm{d} \theta=0,
$$

which gives $E_{j t}(r)=0, r \in(-\infty, 0], j=1,2, \cdots, n$. Therefore, $Z=\left\{P_{0}\right\}$.

Now, using the LaSalle-Lyapunov invariance principle (see [62]), $P_{0}$ is globally stable in $\xi$, if $R_{0} \leq 1$.

\subsection{Global stability of endemic equilibrium}

In this section, we consider that $R_{0}>1$. In this case, it follows from Theorem 4.1 that DFE $P_{0}$ is unstable. Form the uniform persistence results of [63] and similar argument as in the proof of [64, Proposition 3.3], we conclude that instability of $P_{0}$ implies the uniform persistence of system $(2.5)$ in positively invariant set $\bar{\xi}$. This means that, there exists a constant $c(>0)$ such that

$$
\min \left(\liminf _{x \rightarrow 0} S_{k}(t), \liminf _{x \rightarrow 0} E_{k} t(\theta), \liminf _{x \rightarrow 0} U_{k}(t)\right) \geq c \quad \text { for all } k \in \mathbb{N},
$$

provided that $\left(S_{1}(0), E_{10}(\theta), U_{1}(0), \cdots, S_{n}(0), E_{n 0}(\theta), U_{n}(0)\right) \in \bar{\xi}$.

The uniform persistence of model system (2.5) along with boundedness of the solutions in $\bar{\xi}$, implies existence of an endemic equilibrium $P^{*}$ in $\bar{\xi}$, which is summarized in the following proposition.

Proposition 4.1 If $R_{0}>1$, then system (2.5) is uniformly persistent and there exists at least one endemic equilibrium $P^{*}$ in $\bar{\xi}$.

Now, we prove our one main result using Proposition 4.1.

Theorem 4.2 Assume that $B=\left(\beta_{k j}\right)$ is irreducible. If $R_{0}>1$, then system (2.5) has a globally asymptotically stable endemic equilibrium $P^{*}$ in $\bar{\Xi}$.

Proof Let $P^{*}=\left(S_{1}^{*}, E_{1}^{*}, U_{1}^{*}, S_{2}^{*}, E_{2}^{*}, U_{2}^{*}, \cdots, S_{n}^{*}, E_{n}^{*}, U_{n}^{*}\right)$, where $S_{k}^{*}, E_{k}^{*}, U_{k}^{*}>0$ denote the endemic equilibrium of system (2.5). Let $\bar{\beta}_{k j}=\beta_{k j} a_{j} S_{k}^{*} E_{j}^{*}$ and $\bar{B}$ be given by (1.1). Note that $\bar{B}$ is the Laplacian matrix of $\left(\bar{\beta}_{k j}\right)$. Since $B$ is irreducible, $\bar{B}$ is also irreducible. Let $\left\{v_{1}, v_{2}, \cdots, v_{n}\right\}\left(v_{k}>0,1 \leq k \leq n\right)$ be a basis for linear system (1.1) as discussed in Lemma 
1.2. Now, we consider the Lyapunov function to establish the following global stability of endemic equilibrium:

$$
L(t)=L_{1}(t)+L_{2}(t)
$$

where

$$
\begin{aligned}
& L_{1}(t)=\sum_{k=1}^{n} v_{k}\left[S_{k}^{*}\left(\frac{S_{k}}{S_{k}^{*}}-1-\ln \frac{S_{k}}{S_{k}^{*}}\right)+E_{k}^{*}\left(\frac{E_{k}}{E_{k}^{*}}-1-\ln \frac{E_{k}}{E_{k}^{*}}\right)+\frac{b_{k}}{2 e_{k}}\left(U_{k}-U_{k}^{*}\right)^{2}\right], \\
& L_{2}(t)=\sum_{k, j=1}^{n} v_{k} \beta_{k j} S_{k}^{*} \int_{0}^{\infty} a_{j}(\theta) E_{j}^{*}\left(\frac{E_{J}(t-\theta)}{E_{j}^{*}}-1-\ln \frac{E_{J}(t-\theta)}{E_{j}^{*}}\right) \mathrm{d} \theta
\end{aligned}
$$

and

$$
a_{j}(\theta)=\int_{\sigma=\theta}^{\infty} f_{j}(\sigma) \mathrm{d} \sigma .
$$

Since $\psi(x)=x-1-\ln x \geq 0$ for all $x>0$. It is clear that $L(t)$ is always bounded for all $t>0$. $L(t) \geq 0$ and the equality holds if and only if $S_{k}=S_{k}^{*}, E_{k}^{*}=E(t-\theta)=E_{k}^{*}, U_{k}=U_{k}^{*}$. Now, differentiating $L_{1}$ along the solution of system (2.5), we obtain

$$
\begin{aligned}
L_{1}^{\prime}= & \sum_{k=1}^{n} v_{k}\left[S_{k}^{\prime}-\frac{S_{k}^{*}}{S_{k}} S_{k}^{\prime}+E_{k}^{\prime}-\frac{E_{k}^{*}}{E_{k}} E_{k}^{\prime}+\frac{b_{k}}{e_{k}}\left(U_{k}-U_{k}^{*}\right) U_{k}^{\prime}\right] \\
= & \sum_{k=1}^{n} v_{k}\left[\left(1-p_{k}\right) \Gamma_{k}-\left(d_{k}^{s}+\delta_{k}\right) S_{k}-b_{k} U_{k} S_{k}-\left(1-p_{k}\right) \Gamma_{k} \frac{S_{k}^{*}}{S_{k}}+\left(d_{k}^{s}+\delta_{k}\right) S_{k}^{*}\right. \\
& +\sum_{j=1}^{n} \beta_{k j} S_{k}^{*} \int_{0}^{\infty} f_{j}(\theta) E_{j}(t-\theta) \mathrm{d} \theta+b_{k} U_{k} S_{k}^{*}-\left(d_{k}^{E}+\varepsilon_{k}\right) E_{k} \\
& \left.-\frac{E_{k}^{*}}{E_{k}} \sum_{j=1}^{n} \beta_{k j} S_{k} \int_{0}^{\infty} f_{j}(\theta) E_{j}(t-\theta) \mathrm{d} \theta+\left(d_{k}^{E}+\varepsilon_{k}\right) E_{k}^{*}\right] .
\end{aligned}
$$

Using the equilibrium state equation of model system (2.5), we obtain

$$
\begin{aligned}
L_{1}^{\prime}= & \sum_{k=1}^{n} v_{k}\left[\left(d_{k}^{s}+\delta_{k}\right)\left(2-\frac{S_{k}}{S_{k}^{*}}-\frac{S_{k}^{*}}{S_{k}}\right)+b_{k} U_{k}^{*} S_{k}^{*}\left(2-\frac{S_{k}}{S_{k}^{*}}-\frac{S_{k}^{*}}{S_{k}}\right)-\frac{b_{k} f_{k}}{e_{k}}\left(U_{k}-U_{k}^{*}\right)^{2}\right. \\
& +\sum_{j=1}^{n} \beta_{k j} S_{k}^{*} a_{j} E_{j}^{*}\left(2-\frac{S_{k}^{*}}{S_{k}}-\frac{E_{k}}{E_{k}^{*}}\right)+\sum_{j=1}^{n} \beta_{k j} S_{k}^{*} \int_{0}^{\infty} f_{j}(\theta) E_{j}(t-\theta) \mathrm{d} \theta \\
& \left.-\frac{E_{k}^{*}}{E_{k}} \sum_{j=1}^{n} \beta_{k j} S_{k} \int_{0}^{\infty} f_{j}(\theta) E_{j}(t-\theta) \mathrm{d} \theta\right] \\
= & \sum_{k=1}^{n} v_{k}\left[\left(d_{k}^{s}+\delta_{k}+b_{k} U_{k}^{*} S_{k}^{*}\right)\left(2-\frac{S_{k}}{S_{k}^{*}}-\frac{S_{k}^{*}}{S_{k}}\right)-\frac{b_{k} f_{k}}{e_{k}}\left(U_{k}-U_{k}^{*}\right)^{2}\right. \\
& \left.+\sum_{j=1}^{n} \beta_{k j} S_{k}^{*} E_{j}^{*}\left\{a_{j}\left(2-\frac{S_{k}}{S_{k}^{*}}-\frac{S_{k}^{*}}{S_{k}}\right)+\left(\frac{1}{E_{j}^{*}}-\frac{S_{k} E_{k}^{*}}{S_{k}^{*} E_{k} E_{j}^{*}}\right) \int_{0}^{\infty} f_{j}(\theta) E_{j}(t-\theta) \mathrm{d} \theta\right\}\right] .
\end{aligned}
$$


Now, differentiating $L_{2}$ along the solution of model system (2.5) and using the integration by parts, we obtain

$$
\begin{aligned}
L_{2}^{\prime} & =\sum_{k, j=1}^{n} v_{k} \beta_{k j} S_{k}^{*} \frac{\mathrm{d}}{\mathrm{d} t} \int_{0}^{\infty} a_{j}(\theta)\left(E_{j}(t-\theta)-E_{j}^{*}-E_{j}^{*} \ln \frac{E_{j}(t-\theta)}{E_{j}^{*}}\right) \mathrm{d} \theta \\
& =\sum_{k, j=1}^{n} v_{k} \beta_{k j} S_{k}^{*} \int_{0}^{\infty} a_{j}(\theta)\left[-\frac{\partial}{\partial \theta}\left(E_{j}(t-\theta)-E_{j}^{*}-E_{j}^{*} \ln \frac{E_{j}(t-\theta)}{E_{j}^{*}}\right)\right] \mathrm{d} \theta \\
& =\sum_{k, j=1}^{n} v_{k} \beta_{k j} S_{k}^{*} E_{j}^{*}\left(\frac{a_{j} E_{j}}{E_{j}^{*}}-\frac{1}{E_{j}^{*}} \int_{0}^{\infty} f_{j}(\theta) E_{j}(t-\theta) \mathrm{d} \theta-\int_{0}^{\infty} f_{j}(\theta) \ln \frac{E_{j}(t)}{E_{j}(t-\theta)} \mathrm{d} \theta\right) .
\end{aligned}
$$

Now, we calculate $L^{\prime}(t)=L_{1}^{\prime}+L_{2}^{\prime}$,

$$
\begin{aligned}
& L^{\prime}=\sum_{k=1}^{n} v_{k}\left[\left(d_{k}^{s}+\delta_{k}+b_{k} U_{k}^{*} S_{k}^{*}\right)\left(2-\frac{S_{k}}{S_{k}^{*}}-\frac{S_{k}^{*}}{S_{k}}\right)-\frac{b_{k} f_{k}}{e_{k}}\left(U_{k}-U_{k}^{*}\right)^{2}\right. \\
& +\sum_{j=1}^{n} \beta_{k j} S_{k}^{*} E_{j}^{*}\left\{a_{j}\left(2-\frac{S_{k}^{*}}{S_{k}}-\frac{E_{k}}{E_{k}^{*}}-\frac{E_{j}}{E_{j}^{*}}\right)-\int_{0}^{\infty} f_{j}(\theta) \ln \frac{E_{j}(t)}{E_{j}(t-\theta)} \mathrm{d} \theta\right. \\
& \left.\left.-\frac{S_{k} E_{k}^{*}}{S_{k}^{*} E_{k} E_{j}^{*}} \int_{0}^{\infty} f_{j}(\theta) E_{j}(t-\theta) \mathrm{d} \theta\right\}\right] \\
& \leq \sum_{k, j=1}^{n} v_{k} \beta_{k j} S_{k}^{*} E_{j}^{*}\left[a_{j}\left(2-\frac{S_{k}^{*}}{S_{k}}-\frac{E_{k}}{E_{k}^{*}}-\frac{E_{j}}{E_{j}^{*}}\right)-\int_{0}^{\infty} f_{j}(\theta) \ln \frac{E_{j}(t)}{E_{j}(t-\theta)} \mathrm{d} \theta\right. \\
& \left.-\frac{S_{k} E_{k}^{*}}{S_{k}^{*} E_{k} E_{j}^{*}} \int_{0}^{\infty} f_{j}(\theta) E_{j}(t-\theta) \mathrm{d} \theta\right] \\
& =\sum_{k, j=1}^{n} v_{k} \beta_{k j} S_{k}^{*} E_{j}^{*}\left[\int_{0}^{\infty} f_{j}(\theta)\left(2-\frac{S_{k}^{*}}{S_{k}}-\frac{E_{k}}{E_{k}^{*}}-\frac{E_{j}}{E_{j}^{*}}\right)-\int_{0}^{\infty} f_{j}(\theta) \ln \frac{E_{j}(t)}{E_{j}(t-\theta)} \mathrm{d} \theta\right. \\
& \left.-\frac{S_{k} E_{k}^{*}}{S_{k}^{*} E_{k} E_{j}^{*}} \int_{0}^{\infty} f_{j}(\theta) E_{j}(t-\theta) \mathrm{d} \theta\right] \\
& =\sum_{k, j=1}^{n} v_{k} \beta_{k j} S_{k}^{*} E_{j}^{*} \int_{0}^{\infty} f_{j}(\theta)\left[2-\frac{S_{k}^{*}}{S_{k}}-\frac{E_{k}}{E_{k}^{*}}-\frac{E_{j}}{E_{j}^{*}}-\ln \frac{E_{j}(t)}{E_{j}(t-\theta)}-\frac{S_{k} E_{k}^{*}}{S_{k}^{*} E_{k} E_{j}^{*}} E_{j}(t-\theta)\right] \\
& =\sum_{k, j=1}^{n} v_{k} \beta_{k j} S_{k}^{*} E_{j}^{*} \int_{0}^{\infty} f_{j}(\theta)\left[\left(1-\frac{S_{k}^{*}}{S_{k}}+\ln \frac{S_{k}^{*}}{S_{k}}\right)+\left(\frac{E_{j}}{E_{j}^{*}}-\frac{E_{k}}{E_{k}^{*}}\right)-\ln \frac{E_{j} E_{k}^{*}}{E_{j}^{*} E_{k}}\right. \\
& \left.+\left(1-\frac{S_{k} E_{j} E_{k}^{*} E_{j}(t-\theta)}{S_{k}^{*} E_{k} E_{j}^{*}}+\ln \frac{S_{k} E_{j} E_{k}^{*} E_{j}(t-\theta)}{S_{k}^{*} E_{k} E_{j}^{*}}\right)\right] \\
& \leq \sum_{k, j=1}^{n} v_{k} \beta_{k j} S_{k}^{*} E_{j}^{*} \int_{0}^{\infty} f_{j}(\theta)\left[\left(\frac{E_{j}}{E_{j}^{*}}-\frac{E_{k}}{E_{k}^{*}}\right)-\ln \frac{E_{j} E_{k}^{*}}{E_{j}^{*} E_{k}}\right] \\
& =\sum_{k, j=1}^{n} v_{k} \beta_{k j} S_{k}^{*} E_{j}^{*} a_{j}\left[\left(\frac{E_{j}}{E_{j}^{*}}-\frac{E_{k}}{E_{k}^{*}}\right)-\ln \frac{E_{j} E_{k}^{*}}{E_{j}^{*} E_{k}}\right] \\
& =\sum_{k, j=1}^{n} v_{k} \bar{\beta}_{k j}\left[\left(\frac{E_{j}}{E_{j}^{*}}-\frac{E_{k}}{E_{k}^{*}}\right)-\ln \frac{E_{j} E_{k}^{*}}{E_{j}^{*} E_{k}}\right] \text {. }
\end{aligned}
$$


Here, we use the following notation

$$
H_{n}=H_{n}\left(E_{1}, E_{2}, \cdots, E_{n}\right)=\sum_{k, j=1}^{n} v_{k} \bar{\beta}_{k j}\left[\left(\frac{E_{j}}{E_{j}^{*}}-\frac{E_{k}}{E_{k}^{*}}\right)-\ln \frac{E_{j} E_{k}^{*}}{E_{j}^{*} E_{k}}\right] .
$$

Now, we will show that $L^{\prime} \leq 0$ and for this we need to show that $H_{n}=0$. Here we consider the cases $n=1$ and $n=2$ separately and finally $n \geq 3$.

Case-1: If we take $n=1$, then obviously $H_{1}=0$. Then $L^{\prime}(t) \leq 0$ with equality satisfying if and only if $S_{1}(t)=S_{1}^{*}, E_{1}(t)=E_{1}(t-\theta)=E_{1}^{*}, U_{1}(t)=U_{1}^{*}$ for all $t \geq 0, \theta \in\left[0, \theta^{+}\right]$.

Case-2: If we take $n=2$, then $H_{2}=H_{2}\left(E_{1}, E_{2}\right)=\sum_{k, j=1}^{2} v_{k} \bar{\beta}_{k j}\left[\left(\frac{E_{j}}{E_{j}^{*}}-\frac{E_{k}}{E_{k}^{*}}\right)-\ln \frac{E_{j} E_{k}^{*}}{E_{j}^{*} E_{k}}\right]$.

Now, from Lemma 1.2 , we obtain $v_{1}=\bar{\beta}_{21}$ and $v_{2}=\bar{\beta}_{12}$. By expanding $H_{2}$, we have

$$
\begin{aligned}
H_{2} & =v_{1} \bar{\beta}_{12}\left[\left(\frac{E_{2}}{E_{2}^{*}}-\frac{E_{1}}{E_{1}^{*}}\right)-\ln \frac{E_{1} E_{2}^{*}}{E_{1}^{*} E_{2}}\right]+v_{2} \bar{\beta}_{21}\left[\left(\frac{E_{1}}{E_{1}^{*}}-\frac{E_{2}}{E_{2}^{*}}\right)-\ln \frac{E_{1} E_{2}^{*}}{E_{1}^{*} E_{2}}\right] \\
& =\bar{\beta}_{21} \bar{\beta}_{12}\left[\left(\frac{E_{2}}{E_{2}^{*}}-\frac{E_{1}}{E_{1}^{*}}\right)-\ln \frac{E_{2} E_{1}^{*}}{E_{2}^{*} E_{1}}\right]+\bar{\beta}_{12} \bar{\beta}_{21}\left[\left(\frac{E_{1}}{E_{1}^{*}}-\frac{E_{2}}{E_{2}^{*}}\right)-\ln \frac{E_{1} E_{2}^{*}}{E_{1}^{*} E_{2}}\right]
\end{aligned}
$$

(by putting $v_{1}=\bar{\beta}_{21}$ and $v_{2}=\bar{\beta}_{12}$ )

$$
\begin{aligned}
& =\bar{\beta}_{12} \bar{\beta}_{21}\left[-\ln \frac{E_{2} E_{1}^{*}}{E_{2}^{*} E_{1}}-\ln \frac{E_{1} E_{2}^{*}}{E_{1}^{*} E_{2}}\right] \\
& =0 .
\end{aligned}
$$

Hence, $H_{2}=0$ and $L^{\prime}(t) \leq 0$. Equality satisfies if and only if $S_{k}(t)=S_{k}^{*}, E_{k}(t)=E_{k}(t-\theta)=$ $E_{k}^{*}, U_{k}(t)=U_{k}^{*}$, for all $t \geq 0, \theta \in\left[0, \theta^{+}\right]$, where $k=1,2$.

Case-3: Let $n \geq 3$. The function $H_{n}$ becomes complicated. It is difficult to solve it manually. So we will use the graph theoretic technique. Let $H_{n}=H_{n}^{1}+H_{n}^{2}$, where $H_{n}^{1}=$ $\sum_{k, j=1}^{n} v_{k} \bar{\beta}_{k j}\left(\frac{E_{j}}{E_{j}^{*}}-\frac{E_{k}}{E_{k}^{*}}\right)$ and $H_{n}^{2}=\sum_{k, j=1}^{n} v_{k} \bar{\beta}_{k j} \ln \frac{E_{j} E_{k}^{*}}{E_{j}^{*} E_{k}}$. In this case, we first prove that $H_{n}^{1}=$ $\sum_{k, j=1}^{n} v_{k} \bar{\beta}_{k j}\left(\frac{E_{j}}{E_{j}^{*}}-\frac{E_{k}}{E_{k}^{*}}\right)=0$.

From (1.1), we have

$$
v_{k} \sum_{j=1}^{n} \bar{\beta}_{k j}=\sum_{j=1}^{n} \bar{\beta}_{j k} v_{j}, \quad k=1,2, \cdots, n .
$$

By putting $\bar{\beta}_{k j}=\beta_{k j} a_{j} S_{k}^{*} E_{j}^{*}$, we obtain

$$
v_{k} \sum_{j=1}^{n} \beta_{k j} a_{j} S_{k}^{*} E_{j}^{*}=\sum_{j=1}^{n} \beta_{j k} a_{k} S_{j}^{*} E_{k}^{*} v_{j} ; \quad k=1,2, \cdots, n .
$$

Using (4.7), we obtain

$$
\sum_{k, j=1}^{n} v_{k} \bar{\beta}_{k j} \frac{E_{j}}{E_{j}^{*}}=\sum_{k, j=1}^{n} v_{k} \beta_{k j} a_{j} S_{k}^{*} E_{j}=\sum_{k, j=1}^{n} v_{j} \beta_{j k} a_{k} S_{j}^{*} E_{k}
$$




$$
\begin{aligned}
& =\sum_{k, j=1}^{n} \frac{E_{k}}{E_{k}^{*}} v_{k} \beta_{k j} a_{j} S_{k}^{*} E_{j}^{*} \\
& =\sum_{k, j=1}^{n} v_{k} \bar{\beta}_{k j} \frac{E_{k}}{E_{k}^{*}}, \quad \text { where } \beta_{k j} a_{j} S_{k}^{*} E_{j}^{*}=\bar{\beta}_{k j} .
\end{aligned}
$$

Therefore,

$$
\sum_{k, j=1}^{n} v_{k} \bar{\beta}_{k j}\left(\frac{E_{j}}{E_{j}^{*}}-\frac{E_{k}}{E_{k}^{*}}\right)=0 \text { holds for } E_{1}, E_{2}, \cdots, E_{n}>0
$$

Now, we show that $H_{n}^{2}=0$, i.e., $\sum_{k, j=1}^{n} v_{k} \bar{\beta}_{k j} \ln \frac{E_{j} E_{k}^{*}}{E_{j}^{*} E_{k}}=0$ holds for $E_{1}, E_{2}, \cdots, E_{n}>0$.

By Lemma 1.2, $v_{k}=K_{k k}$ is a sum of all rooted directed spanning subtrees $T$ of $G$ of root at vertex $k$. If we add a directed $\operatorname{arc}(k, j)$ from root vertex $k$ to another vertex $j$, then we get a unicyclic subgraph $X$ of $G$ and each term $v_{k} \bar{\beta}_{k j}$ is the weight $w(X)$ of unicyclic subgraph $X$. Further, we observe that the $\operatorname{arc}(k, j)$ is an arc of the unique cycle $C X$ of $X$. Moreover, we can form the same unicylic $X$ by adding each arc of $C X$ to corresponding tree $T$. Thus, the meaning of double sum (over $k$ and $j$ ) in $H_{n}^{2}$ can be considered as a sum over all the arcs in the cycle of all the unicyclic subgraph $H$ containing vertices $\{1,2, \cdots, n\}$ of graph $G$, that is

$$
\begin{aligned}
H_{n}^{2} & =\sum_{X} H_{n, X}^{2}=\sum_{X} w(X) . \sum_{(k, j) \in E(C X)} \ln \frac{E_{j} E_{k}^{*}}{E_{j}^{*} E_{k}} \\
& =\sum_{X} w(X) \cdot \ln \left(\prod_{(k, j) \in E(C X)} \frac{E_{j} E_{k}^{*}}{E_{j}^{*} E_{k}}\right)
\end{aligned}
$$

where $E(C X)$ is the set of all arcs of unique cycle $C X$. Then, we have

$$
\prod_{(k, j) \in E(C X)} \frac{E_{j} E_{k}^{*}}{E_{j}^{*} E_{k}}=1, \quad \text { therefore, } \quad \ln \left(\prod_{(k, j) \in E(C X)} \frac{E_{j} E_{k}^{*}}{E_{j}^{*} E_{k}}\right)=0 .
$$

Thus $H_{n, X}^{2}=0$ for each unicylic subgraph $X$. For example, let $n=2$. The unique cycle $C X$ has two vertices $\{1,2\}$ and makes a cycle $1 \rightarrow 2 \rightarrow 1$. Here $E(C X)=\{(1,2),(2,1)\}$ and

$$
\prod_{(k, j) \in E(C X)} \frac{E_{j} E_{k}^{*}}{E_{j}^{*} E_{k}}=\frac{E_{2} E_{1}^{*}}{E_{2}^{*} E_{1}} \cdot \frac{E_{1} E_{2}^{*}}{E_{1}^{*} E_{2}}=1 .
$$

Then we have $H_{n}^{2}=\sum_{X} H_{n, X}^{2}=0$ holds for $E_{1}, E_{2}, \cdots, E_{n}>0$, which gives that $H_{n}=0$ for $E_{1}, E_{2}, \cdots, E_{n}>0$.

Therefore, $L^{\prime} \leq 0$ for all $\left(S_{1}, E_{1}, U_{1}, \cdots, S_{n}, E_{n}, U_{n}\right) \in \bar{\xi}$ and equality holds if and only if $S_{k}(t)=S_{k}^{*}, E_{k}(t)=E_{k}(t-\theta)=E_{k}^{*}, U_{k}=U_{k}^{*}$ and $H_{n}=0$. Hence, we conclude that the only invariant set of system $(2.5)$ in $\left\{\left(S_{k}, E_{K}, U_{k}\right) \in \bar{\xi}: \frac{\mathrm{d} L}{\mathrm{~d} t}=0\right\}$ is the endemic equilibrium $P^{*}$. Thus, if $R_{0}>1$, then $P^{*}$ is globally asymptotically stable and unique in the $\bar{\xi}$-region (LaSalle's Invariance Principle, see [62]). 


\section{Numerical Simulations}

In this section, we show the feasibility of our main theoretical results. We discuss the effects of feedback variables and infection age on the transmission of the infectious disease. For the simplicity of our model, we consider an example with $n=2$ for numerical simulation to support our main results.

$$
\begin{aligned}
\frac{\mathrm{d} S_{k}}{\mathrm{~d} t} & =\left(1-p_{k}\right) \Gamma_{k}-\left(d_{k}^{S}+\delta_{k}\right) S_{k} \\
- & \sum_{j=1}^{n} \beta_{k j} S_{k} \int_{0}^{\infty} h_{j}(\theta) i_{j}(t, \theta) \mathrm{d} \theta-b_{k} U_{k} S_{k}, \\
\frac{\mathrm{d} E_{k}}{\mathrm{~d} t} & =\sum_{j=1}^{n} \beta_{k j} S_{k} \int_{0}^{\infty} h_{j}(\theta) i_{j}(t, \theta) \mathrm{d} \theta-\left(d_{k}^{E}+\varepsilon_{k}\right) E_{k}, \quad \text { where } k=1,2 . \\
\left.\frac{\left(\frac{\partial}{\partial t}\right.}{}+\frac{\partial}{\partial \theta}\right) i_{k}(t, \theta)=-\left(d_{k}^{I}+\gamma_{k}\right) i_{k}(t, \theta), & \\
\frac{\mathrm{d} U_{k}}{\mathrm{~d} t} & =-f_{k} U_{k}+e_{k} S_{k}, \\
\frac{\mathrm{d} R_{k}}{\mathrm{~d} t} & =p_{k} \Gamma_{k}+\delta_{k} S_{k}+\gamma_{k} \int_{0}^{\infty} i_{k}(t, \theta) \mathrm{d} \theta-d_{k}^{R} R_{k},
\end{aligned}
$$

A numerical example of associated $0 \mathrm{DE}$ model system with $n=2$ would be given

$$
\begin{array}{rlr}
\frac{\mathrm{d} S_{k}}{\mathrm{~d} t} & =\left(1-p_{k}\right) \Gamma_{k}-\left(d_{k}^{S}+\delta_{k}\right) S_{k}-\sum_{j=1}^{n} \beta_{k j} S_{k} I_{j}-b_{k} U_{k} S_{k}, & \\
\frac{\mathrm{d} E_{k}}{\mathrm{~d} t} & =\sum_{j=1}^{n} \beta_{k j} S_{k} I_{j}-\left(d_{k}^{E}+\varepsilon_{k}\right) E_{k}, & \\
\frac{\mathrm{d} I_{k}}{\mathrm{~d} t} & =\varepsilon_{k} E_{k}-\left(d_{k}^{I}+\gamma_{k}\right) I_{k}, & \\
\frac{\mathrm{d} U_{k}}{\mathrm{~d} t} & =-f_{k} U_{k}+e_{k} S_{k}, & \\
\frac{\mathrm{d} R_{k}}{\mathrm{~d} t} & =p_{k} \Gamma_{k}+\delta_{k} S_{k}+\gamma_{k} I_{k}-d_{k}^{R} R_{k}, &
\end{array}
$$

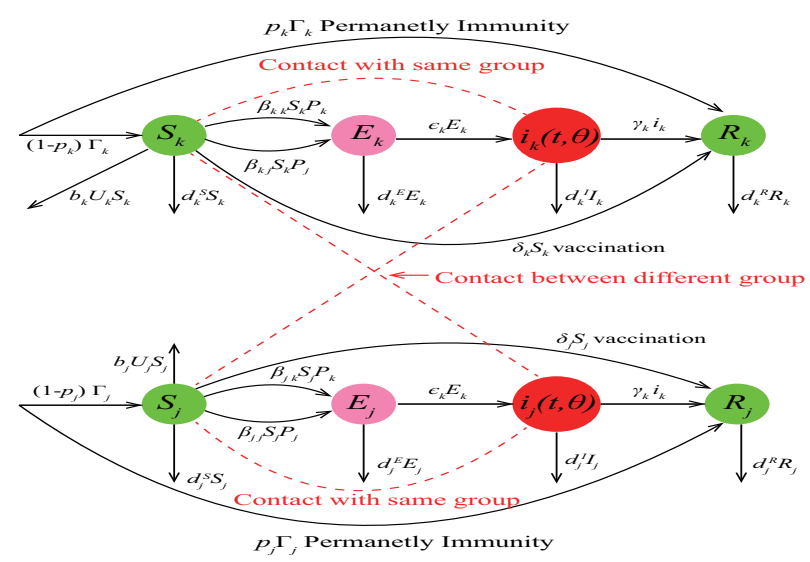

Figure 1 The transfer diagram for model system (2.1), where $P_{j}=\int_{0}^{\infty} h_{j}(\theta) i_{j}(t, \theta) \mathrm{d} \theta$ and $P_{k}=\int_{0}^{\infty} h_{k}(\theta) i_{k}(t, \theta) \mathrm{d} \theta$. The red line is corresponding to the transmission process. 
In Figure 1, we show the disease transmission diagram of model system (3).

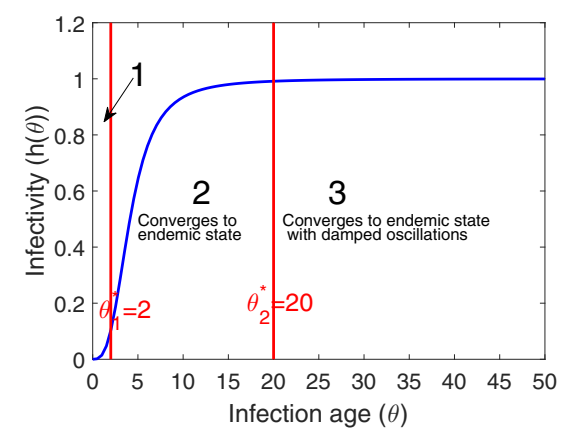

Figure 2 The graph shows changes in infectivity of infected individuals (for both groups) with respect to infection age.

In Figure 2, we describe the infectivity of infected individuals for varying infection age. Here we observe that the infectivity increases and becomes saturated after a threshold of infection age. This situation can arise to a disease which becomes more and more transmissible with increasing infection age. This type of infectivity function could be applicable to Ebola disease (see [27]).

Table 1 Model parameters and their definitions.

\begin{tabular}{cl}
\hline Parameters & Definition \\
\hline$d_{k}^{s}$ & Natural death rate of susceptible individuals in the $k$-th group. \\
$d_{k}^{E}$ & Natural death rate of exposed individuals in the $k$-th group. \\
$d_{k}^{I}$ & Natural death rate of infected individuals in the $k$-th group. \\
$d_{k}^{R}$ & Natural death rate of recovered individuals in the $k$-th group. \\
$p_{k}$ & Fraction of new individuals into the $k$-th group who are \\
& immuned. \\
$\delta_{k}$ & Vaccination rate of susceptible individuals in the $k$-th group. \\
$\gamma_{k}$ & Recovery rate of infected individuals in the $k$-th group. \\
$\varepsilon_{k}$ & Disease induced death rate of infected individuals in the $k$-th \\
& group. \\
$b_{k}, f_{k} e_{k}$ & Feedback parameters.
\end{tabular}

Table 2 Numerical values of parameters.

\begin{tabular}{|c|c|c|c|c|c|}
\hline Parameters & Numerical Value & Units & Parameters & Numerical Value & Units \\
\hline$P_{1}$ & 0.05 & unit less & $P_{2}$ & 0.05 & unit less \\
$\Gamma_{1}$ & 200 & per day & $\Gamma_{2}$ & 250 & per day \\
$\beta_{11}$ & $8 \times 10^{-4}$ & per day & $\beta_{12}$ & $7 \times 10^{-4}$ & per day \\
$\beta_{21}$ & $8 \times 10^{-4}$ & per day & $\beta_{22}$ & $8 \times 10^{-4}$ & per day \\
$\gamma_{1}$ & $5 \times 10^{-6}($ For $(5.1))$ & per day & $\gamma_{1}$ & $51 \times 10^{-7}($ For (5.1)) & per day \\
& 0.5 (For (5.2)) & & & $0.51($ For $(5.2))$ & \\
\hline
\end{tabular}

Figure 3 shows the long-time dynamics of our considered model (5.1) which supports our main mathematical results. Here we observe that the solution curves converge to the diseasefree equilibrium for $R_{0} \leq 1$ (see Figure $3 \mathrm{a}$ ). If $R_{0}>1$, then the solution curves converge to endemic state of disease (see Figure $3 \mathrm{~b}$ ). Thus, Figure 3 also ensures the global stability of the both equilibria of our model system (5.1). 

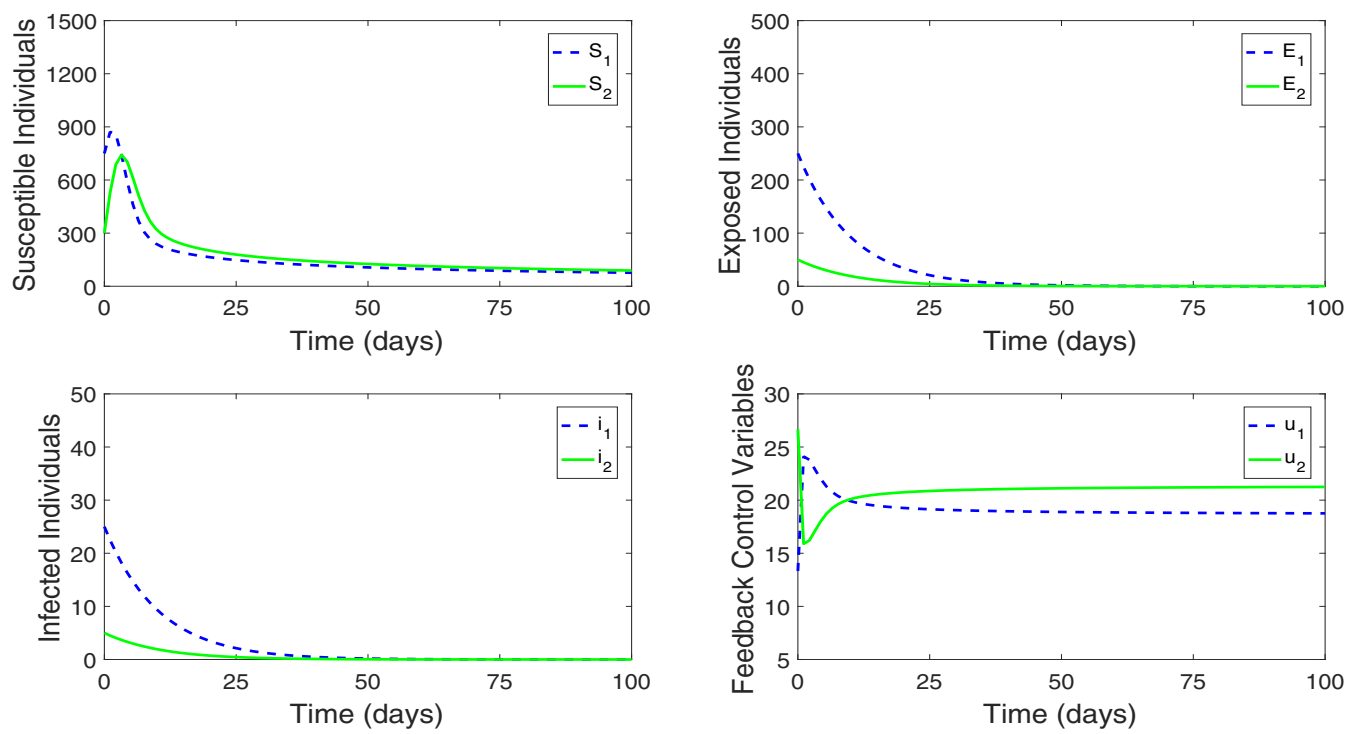

(a) For $R_{0}<1$, the solutions of system (5.1) converge to disease free equilibrium.
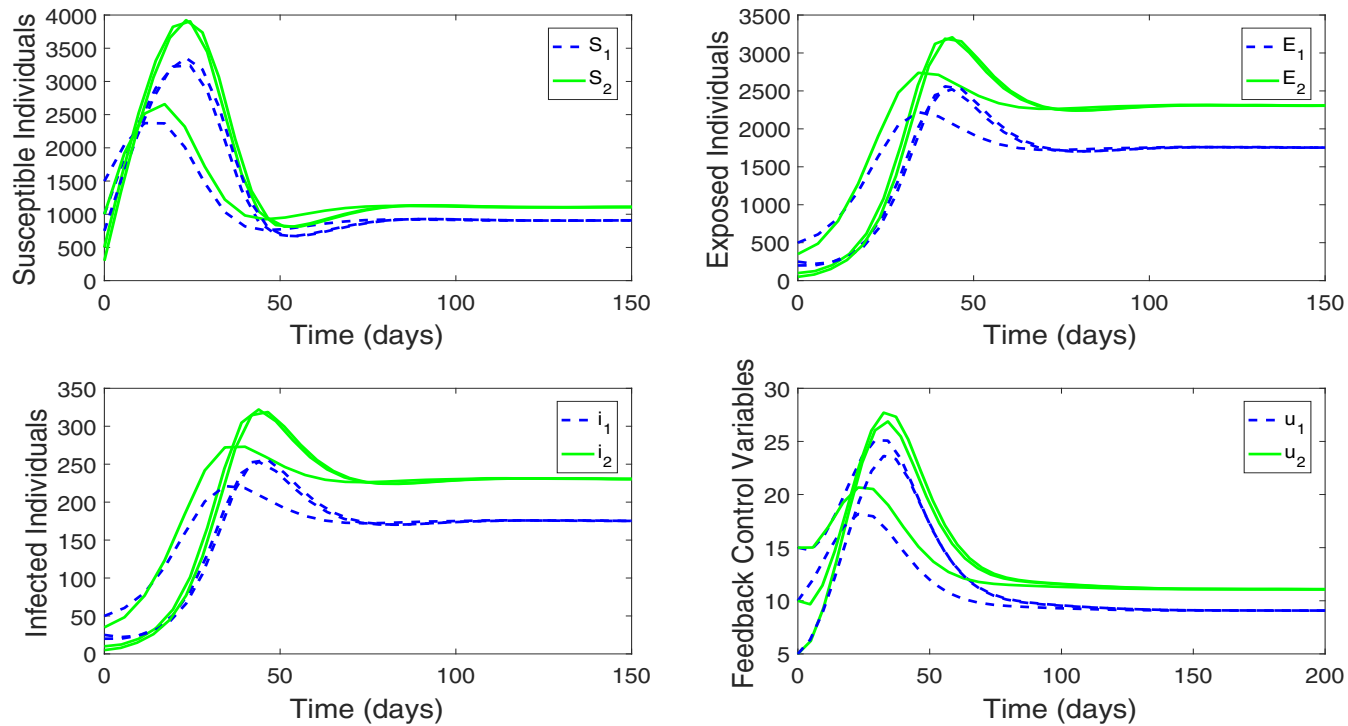

(b) For $R_{0}>1$, the solutions of system converge to endemic state of disease.

Figure 3 The graphs show the long time dynamics of our model system (5.1). Here, blue dashed curves stand for group-1 and green solid curves for group-2. Numerical values of all parameters are given in Table 1. 
In Figure 4a, we observe that if we take the infection age from Region-2 $(2<\theta<20)$ of Figure 2, then the infected population of that infection age converges to endemic state of disease (after reaching peak point). For Region-1 $(\theta<2)$ and Region-3 $(\theta>20)$ of Figure 2, the infected population converges with damped oscillations to the endemic state of disease. In Figure $4 \mathrm{~b}$, we easily observe that in the infection age model, damped oscillations become visible but the associated ODE model system (5.2) does not show any such oscillations.

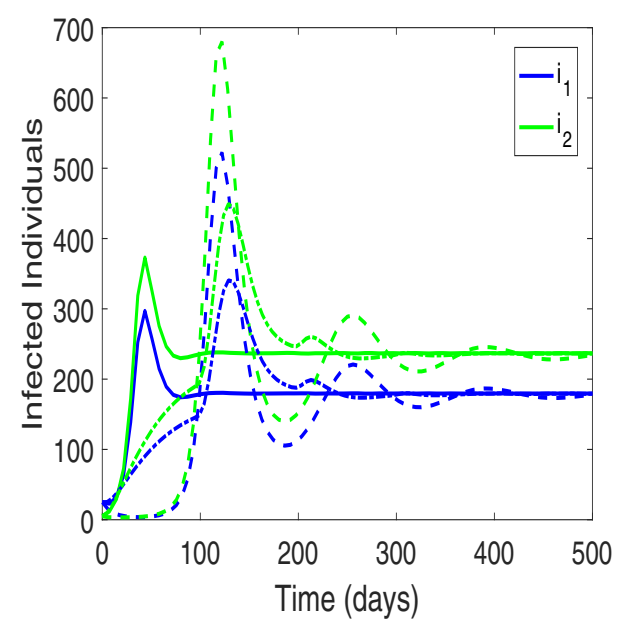

(a) The graph shows the effects of infection age on long time dynamics of infected populations $\left(i_{1}(t, \theta)\right.$ and $\left.i_{2}(t, \theta)\right)$, where dash-dotted curve for $\theta=50$, soild curve for $\theta=5$ and dashed curve for $\theta=1.9$. For all three curves, $f_{1}=f_{2}=e_{1}=e_{2}=$ $b_{1}=b_{2}=0$.

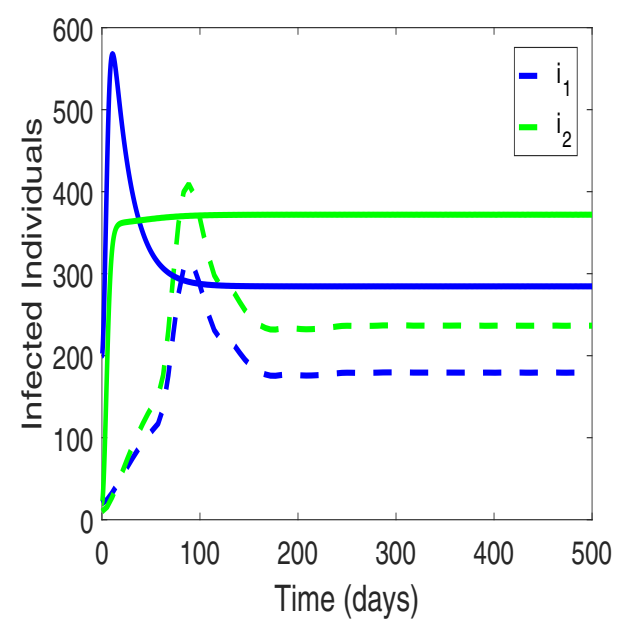

(b) The graph shows the effects of age of infection on long time dynamics of infected populations. Here, dashed curves stand for infection age $(\theta=50)$ of model system (5.1) and solid curves for associated ODE model system (5.2).

Figure 4 The graphs show the effects of age of infection on long time dynamics of infected populations, where numerical values of all parameters are given in Table 2.

Moreover, the effects of four feedback parameters on infected population are compared in Figure 5. According to the gradient of $I$, we rank the effect of parameters as follows: $e_{2}>f_{1}>f_{2}>e_{1}$. It is also found that almost all increases in $e_{i}$ or $f_{i}$ will increase the total number of infections up except $e_{1}$. In the first row of Figure $5, e_{1}$ is labeled on the Y-axis, $I$ will decrease with $e_{1}$ rising. Meanwhile we probe the effecst of $e_{i}$ and $f_{i}$ on each group $I_{1}$ and $I_{2}$. We find that the parameters play the same role on each group on $I$ but different on $I_{2}$ as $e_{1}$ and $f_{1}$ vary. Two groups, $I_{1}$ and $I_{2}$, receive the opposite effects from $e_{1}$ and $f_{1}$. Specifically, when $e_{1}$ descends or $f_{1}$ rises, the number of $I_{1}$ increases but $I_{2}$ show a decline which is shown in Figure 6. Interestingly, direction of gradient change in density completely turns around from an intuitive view. High-density diseased area for $I_{1}$ is low-density diseased area for $I_{2}$, similarly, the low-density diseased area for $I_{1}$ is high-density diseased area for $I_{2}$.

There are some deductions. It may be a suitable method to modulate feedback parameters with obvious effects like $e_{2}$ and $f_{1}$, when we intend to control the total infected population. However, when we want to restrict one of infected groups, both proper parameters and appropriate values must be deliberately selected because the influence over another group can not be neglected. 
(A)

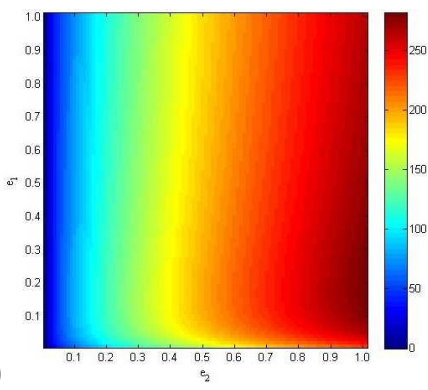

(B)

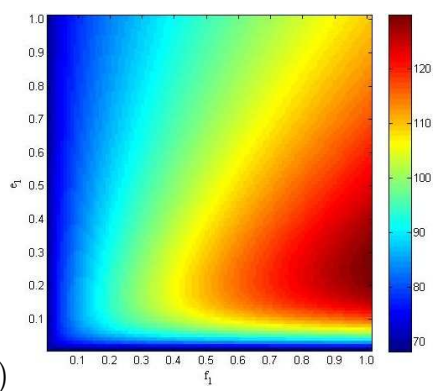

$(\mathrm{C})$

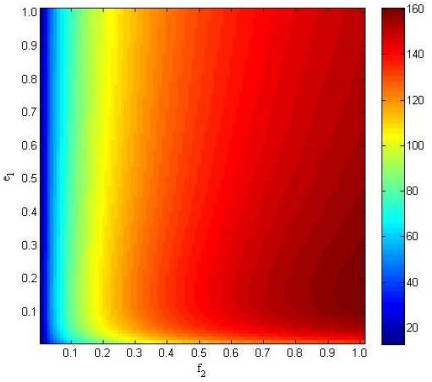

(D)

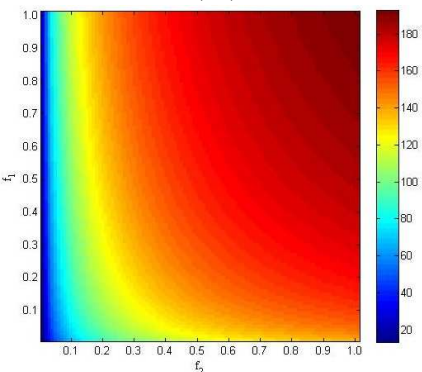

(E)

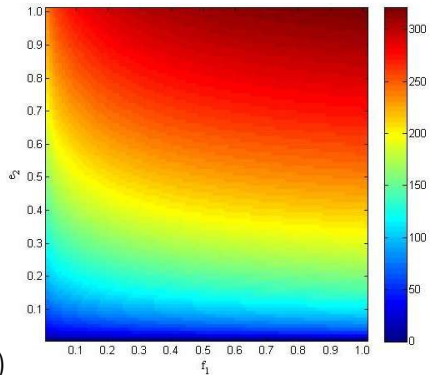

(F)

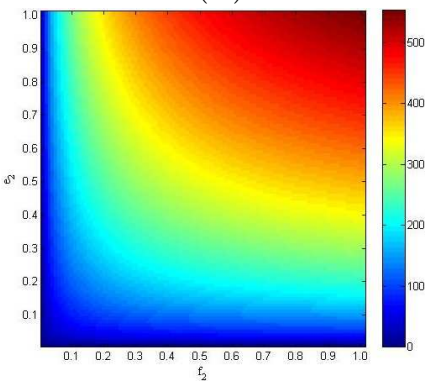

Figure 5 The graphs show the total number of infected population with respect to different values of $e_{i}$ or $f_{i}$. We use $I$ to represent the total number, which is equal to the sum of $I_{1}$ and $I_{2}$. Expect feedback parameters, other parameters are taken from Table 2. (A) $e_{1}$ and $e_{2}$ with $f_{1}=f_{2}=0.1$; (B) $e_{1}$ and $f_{1}$ with $e_{2}=f_{2}=0.1$; (C) $e_{1}$ and $f_{2}$ with $e_{2}=f_{1}=0.1$; (D) $f_{1}$ and $f_{2}$ with $e_{1}=e_{2}=0.1$; (E) $e_{2}$ and $f_{1}$ with $e_{1}=f_{2}=0.1 ;(\mathrm{F}) e_{2}$ and $f_{2}$ with $e_{1}=f_{1}=0.1$. 
(A)
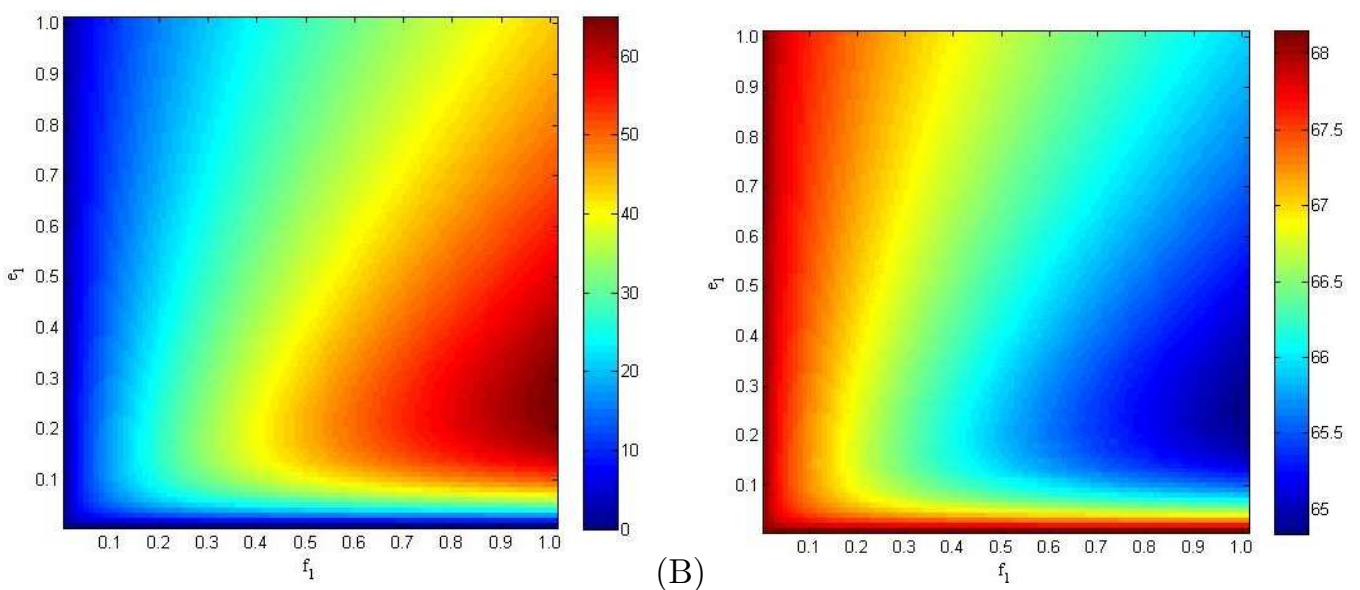

Figure 6 The graphs show the number of each group $I_{1}$ in (A) and $I_{2}$ in (B) with different value of $e_{1}$ and $f_{1}$, corresponding to the total number $I$ shown in Figure 5(B) in which $e_{2}$ and $f_{2}$ are equal to 0.1. The red area in (A) turns blue in (B), blue area in (A) turns red in (B).

The Figure 7 represents the region of feedback parameters, in which $R_{0} \leq 1$ or the disease remains eradicated. The Figure 7 also establishes some thresholds of feedback parameters to the eradication of disease from all considered groups.

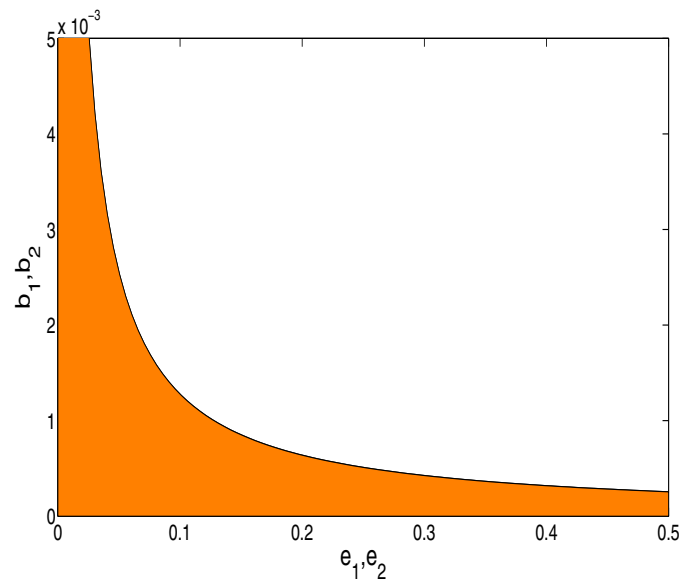

Figure 7 The graph shows the region (shadowed) for $R_{0} \leq 1$, where $f_{1}=f_{2}=5 \times 10^{-10}$ and numerical values of all other parameters are given in Table 2 .

\section{Conclusion}

Over the last few decades, the spread of different infectious diseases (both incurable diseases like HIV/AIDS and major diseases like Cholera) are posing continuous threatening to public health. More than fifty thousands men, women and children are dying everyday due to different kind of infectious diseases. At regular time periods, different strategies are being suggested to 
control the transmission of a particular disease, however, success and failure of such control strategies depend on various factors e.g., heterogeneity of host population. Multi-group approach of mathematical formulation of a particular infectious disease is one of different ways to incorporate the associated heterogeneity in the epidemic system. In this paper, a multi-group SEIR epidemic model system (2.1) incorporating infection age and feedback variables has been studied. The proposed model system (2.1) describes the transmission dynamics of the disease in a heterogeneous host population and via heterogeneity, the irregularity of infectiveness of infectious individuals have been incorporated. Both infection age and feedback have important influence on transmission dynamics of infectious diseases. The main contributions of our study are the following:

(a) The feedback strategy to control the infectious disease, is introduced into an SEIR multi-group epidemic model in which the effects of infection age are considered.

(b) The global stability of endemic equilibrium using some important graph theoretic results to Lyapunov function method has been established.

(c) The numerical simulations of a 2-group example show the influences of feedback and infection age on the dynamics of our proposed model.

Basic reproduction number $R_{0}$ has been computed using the spectral radius of next generation matrix. It is found that the global behaviour of proposed multi-group model system is completely determined via $R_{0}$. More precisely, we show that if $R_{0} \leq 1$, then the disease dies out from all groups that means DFE is globally asymptotically stable. Further, we have also proved that if $R_{0}>1$, then the disease becomes endemic in all the groups. In this way, for $R_{0}>1$, the global asymptotic stability of endemic equilibrium has been established using graph theoretical approach to the method of Lyapunov function. Further, via numerical simulations of a 2-group model system incorporating variable infectivity (infection age), we establish that the initial dynamics of system are very sensitive to the shape and timing of the first prevalence peak, but the long-term dynamics shows the same qualitative behaviour. That means the steady state of each infected individual approaches to almost the same endemic equilibrium. Thieme et al. [29] elaborated that undamped oscillations may also occur if the infectivity is at sufficiently higher level but our results show that the damped oscillations also occur in the initial dynamics of infected individuals. Therefore, this result is the answer of question in special case of work [29]. We have also found that the feedback not only changes the level of endemicity of disease but also can play a major role in the eradication of the disease from all considered groups. This particular result is consistent with result obtained in [43]. The feedback parameters have been ranked in order of effect on total number of infected population. This can provide a strategy to modulate the infected population: Priority to alter the parameter with strong effect is a simple way for a greater change in infected population. The region of feedback parameters has been described where the disease dies out from all groups. Furthermore, it is found that some of feedback parameters have dissimilar effects on different groups. When the overall infected population is adjusted by the control parameters, each group also needs to be considered. This requires special attention in practice. We have also quantified thresholds of infection age with respect to change of the dynamical behaviour of infected individuals and thresholds of the 
feedback in respect of eradication of infectious disease.

Our findings would necessarily contribute for more deeper understanding of role of feedback in the dynamics of infected individuals with infection age. This particular study may also provide important information for future modeling efforts in predicting future epidemics and establishing control strategies. In this way, the findings of this paper may be valuable for health policymakers who work on various types of suitable policies for controlling respective infectious diseases. Moreover, it may be interesting and more reasonable to further investigate of our proposed model by incorporating the death rate and removal rate of infected individuals and taking into account the function of infection age. The threshold dynamics of infected individuals can be investigated and that may change the global stability of equilibria into oscillations. The model system may undergo a Hopf bifurcation. We leave these ideas for future studies.

\section{References}

[1] Oaks, J. S. C., Shope, R. E. and Lederberg, J., Emerging infections: Microbial Threats to Health in the United States, National Academies Press, New York, 1992.

[2] Brauer, F. and Castillo-Chavez, C., Mathematical Models in Population Biology and Epidemiology, Text in Applied Mathematics, 40, Springer-Verlag, New York, 2001.

[3] Gubler, D. J., Resurgent vector-borne diseases as a global health problem, Emerg. Infect. Dis., 4, 1998, 442-450.

[4] Mcnicoll, R. B. G., The World Health Report 1996: Fighting Disease, Fostering Development; Report of the Director-General. by World Health Organization, Population and Development Review, 23, 1997, 203-204.

[5] Anderson, R. M., Anderson, B. and May, R. M., Infectious Diseases of Humans: Dynamics and Control, Oxford University Press, New York, 1992.

[6] Zhang, Z., Kundu, S., Tripathi, J. P. and Bugalia, S., Stability and Hopf bifurcation analysis of an SVEIR epidemic model with vaccination and multiple time delays, Chaos Solitons Fractals, 131, 2020, 109483.

[7] Hethcote, H. W. and Driessche, P. V. D., Some epidemiological models with nonlinear incidence, J. Math. Biol., 29, 1991, 271-287.

[8] Kermack, W. O. and McKendrick, A. G., A contribution to the mathematical theory of epidemics, Proceedings of the royal society of london., Series A, 115, 1927, 700-721.

[9] Hethcote, H. W., Stech, H. W. and Driessche, P. V. D., Periodicity and stability in epidemic models: A survey, Differential Equations and Applications in Ecology, Epidemics, and Population Problems, Elsevier Academies Press, New York-London, 1981, 65-82.

[10] Sun, G. Q., Jusup, M., Jin, Z., et al., Pattern transitions in spatial epidemics: Mechanisms and emergent properties, Phys. Life Rev., 19, 2016, 43-73.

[11] Li, L., Patch invasion in a spatial epidemic model, Appl. Math. Comput., 258, 2015, 342-349.

[12] Guo, Z. G., Song, L. P., Sun, G. Q., et al., Pattern dynamics of an SIS epidemic model with nonlocal delay, Int. J. Bifurcation Chaos, 29, 2019, 1950027.

[13] Sun, G. Q., Wang, C. H., Chang, L. L., et. al., Effects of feedback regulation on vegetation patterns in semi-arid environments, Appl. Math. Model., 61, 2018, 200-215.

[14] Andersson, H. and Britton, T., Heterogeneity in epidemic models and its effect on the spread of infection, J. Appl. Probab., 35, 1998, 651-661.

[15] Bowden, S. and Drake, J., Ecology of multi-host pathogens of animals, Nat Education Knowledge, 4, 2013, $5-5$.

[16] Anderson, R. M. and May, R. M., Spatial, temporal, and genetic heterogeneity in host populations and the design of immunization programmes, Math. Med. Biol., 1, 1984, 233-266.

[17] Luo, X., Yang, J., Jin, Z. and Li, J., An edge-based model for nonMarkovian sexually transmitted infections in coupled network, Int. J. Biomath., 13, 2020, 2050014.

[18] Lajmanovich, A. and Yorke, J. A., A deterministic model for gonorrhea in a nonhomogeneous population, Math. Biosci., 28, 1976, 221-236. 
[19] Li, M. T., Sun, G. Q., Zhang, J. and Jin, Z., Global dynamic behavior of a multigroup cholera model with indirect transmission, Discrete Dyn. Nat. Soc., 2013, 2013, 703826.

[20] Hyman, J. M., Li, J. and Stanley, E. A., The differential infectivity and staged progression models for the transmission of HIV, Math. Biosci., 155, 1999, 77-109.

[21] Beretta, E. and Capasso, V., Global stability results for a multigroup SIR epidemic model, T. G. Hallam, L. J. Gross, S. A. Levin (Eds.), Mathematical Ecology, World Scientific, Singapore, 1986, 317-342.

[22] Hethcote, H. W., An immunization model for a heterogeneous population, Theor. Popul. Biol., 14, 1978, 338-349.

[23] Huang, W., Cooke, K. L. and Castillo-Chavez, C., Stability and bifurcation for a multiple-group model for the dynamics of HIV/AIDS transmission, SIAM J. Appl. Math., 52, 1992, 835-854.

[24] Lin, X. and So, W. H., Global stability of the endemic equilibrium and uniform persistence in epidemic models with subpopulations, J. Aust. Math. Soc., 34, 1993, 282-295.

[25] Thieme, H. R., Local stability in epidemic models for heterogeneous populations, Math. Med. Biol., 97, 1985, 185-189.

[26] Thieme, H. R., Mathematics in Population Biology, Princeton University Press, Princeton, 2018.

[27] Magal, P., McCluskey, C. and Webb, G., Lyapunov functional and global asymptotic stability for an infection-age model, Appl. Anal., 89, 2010, 1109-1140.

[28] Feng, Z., Huang, W. and Castillo-Chavez, C., On the role of variable latent periods in mathematical models for tuberculosis, J. Dyn. Differ. Equ., 13, 2001, 425-452.

[29] Thieme, H. R. and Castillo-Chavez, C., How may infection-age-dependent infectivity affect the dynamics of HIV/AIDS?, SIAM J. Appl. Math., 53, 1993, 1447-1479.

[30] Inaba, H. and Sekine, H., A mathematical model for Chagas disease with infection-age-dependent infectivity, Math. Biosci., 190, 2004, 39-69.

[31] Alexander, M. E., Moghadas, S. M., Röst, G. and Wu, J., A delay differential model for pandemic influenza with antiviral treatment, Bull. Math. Biol., 70, 2008, 382-397.

[32] Röst, G., SEIR epidemiological model with varying infectivity and infinite delay, Math. Biosci. Eng., 5, 2008, 389-402.

[33] McCluskey, C. C., Global stability for an SEIR epidemiological model with varying infectivity and infinite delay, Math. Biosci. Eng., 6, 2009, 603-610.

[34] Li, M. Y., Shuai, Z. and Wang, C., Global stability of multi-group epidemic models with distributed delays, J. Math. Anal. Appl., 361, 2010, 38-47.

[35] Chen, F., Positive periodic solutions of neutral Lotka-Volterra system with feedback control, Appl. Math. Comput., 162, 2005, 1279-1302.

[36] Chen, F., Yang, J., Chen, L. and Xie, X., On a mutualism model with feedback controls, Appl. Math. Comput., 214, 2009, 581-587.

[37] Fan, M., Wang, K., Wong, P. J. and Agarwal, R. P., Periodicity and stability in periodic n-species LotkaVolterra competition system with feedback controls and deviating arguments, Acta Mathematica Sinica, 19, 2003, 801-822.

[38] Gopalsamy, K. and Weng, P. X., Feedback regulation of logistic growth, International Journal of Mathematics and Mathematical Sciences, 16, 1993, 177-192.

[39] Li, Z., Han, M. and Chen, F., Influence of feedback controls on an autonomous Lotka-Volterra competitive system with infinite delays, Nonlinear Anal.-Real World Appl., 14, 2013, 402-413.

[40] Niyaz, T. and Muhammadhaji, A., Positive periodic solutions of cooperative systems with delays and feedback controls, International Journal of Differential Equations, 2013, 2013, 502963.

[41] Xiao, Y. N., Tang, S. Y. and Chen, J. F., Permanence and periodic solution in competitive system with feedback controls, Math. Comput. Model., 27, 1998, 33-37.

[42] Fan, Y. H. and Wang, L. L., Global asymptotical stability of a Logistic model with feedback control, Nonlinear Anal.-Real World Appl., 11, 2010, 2686-2697.

[43] Yang, K., Miao, Z., Chen, F. and Xie, X., Influence of single feedback control variable on an autonomous Holling-II type cooperative system, J. Math. Anal. Appl., 435, 2016, 874-888.

[44] Li, H. L., Zhang, L., Teng, Z., et al., Global stability of an SI epidemic model with feedback controls in a patchy environment, Appl. Math. Comput., 321, 2018, 372-384.

[45] Sun, R., Global stability of the endemic equilibrium of multigroup SIR models with nonlinear incidence, Comput. Math. Appl., 60, 2010, 2286-2291. 
[46] Shang, Y., Global stability of disease-free equilibria in a two-group SI model with feedback control, Nonlinear Anal. Model. Control, 20, 2015, 501-508.

[47] Tripathi, J. P. and Abbas, S., Global dynamics of autonomous and nonautonomous SI epidemic models with nonlinear incidence rate and feedback controls, Nonlinear Dyn., 86, 2016, 337-351.

[48] Guo, H., Li, M. Y. and Shuai, Z., Global stability of the endemic equilibrium of multigroup SIR epidemic models, Can. Appl. Math. Q., 14, 2006, 259-284.

[49] Guo, H., Li, M. and Shuai, Z., A graph-theoretic approach to the method of global Lyapunov functions, Proc. Amer. Math. Soc., 136, 2008, 2793-2802.

[50] Kuniya, T., Global stability of a multi-group SVIR epidemic model, Nonlinear Anal.-Real World Appl., 14, 2013, 1135-1143.

[51] Li, M. Y. and Shuai, Z., Global-stability problem for coupled systems of differential equations on networks, J. Differ. Equ., 248, 2010, 1-20.

[52] Shuai, Z. and Driessche, P. V. D., Global stability of infectious disease models using lyapunov functions, SIAM J. Appl. Math., 73, 2013, 1513-1532.

[53] Li, M. T., Jin, Z., Sun, G. Q. and Zhang, J., Modeling direct and indirect disease transmission using multi-group model, J. Math. Anal. Appl., 446, 2017, 1292-1309.

[54] Shuai, Z. S. and Driessche, P. V. D., Global dynamics of cholera models with differential infectivity, Math. Biosci., 234, 2011, 118-126.

[55] Horn, R. A. and Johnson, C. R., Matrix Analysis, Cambridge university press, Cambridge, 2012.

[56] Moon, J. W., Counting Labelled Trees, Canadian Mathematical Congress, Montreal, 1970.

[57] Berman, A. and Plemmons, R. J., Nonnegative Matrices in the Mathematical Sciences, Academic Press, New York, 1994.

[58] FV, A. and Haddock, J., On determining phase spaces for functional differential equations, Funkcialaj Ekvacioj, 31, 1988, 331-347.

[59] Kolmanovskii, V. and Myshkis, A., Applied theory of functional differential equations, Mathematics and its Applications (Soviet Series), 85. Kluwer Academic Publishers Group, Dordrecht, 1992.

[60] Birkhoff, G. and Rota, G. C., Ordinary Differential Equations, John Wiley \& Sons, New York, 1989.

[61] Diekmann, O., Heesterbeek, J. A. P. and Metz, J. A., On the definition and the computation of the basic reproduction ratio $R_{0}$ in models for infectious diseases in heterogeneous populations, J. Math. Biol., 28, 1990, 365-382.

[62] LaSalle, J. P., The stability of dynamical systems, Regional Conference Series in Applied Mathematics. Society for Industrial and Applied Mathematics, Philadelphia, 1976.

[63] Freedman, H. I., Ruan, S. and Tang, M., Uniform persistence and flows near a closed positively invariant set, J. Differ. Equ., 6, 1994, 583-600.

[64] Li, M. Y., Graef, J. R., Wang, L. and Karsai, J., Global dynamics of a SEIR model with varying total population size, Math. Biosci., 160, 1999, 191-213. 